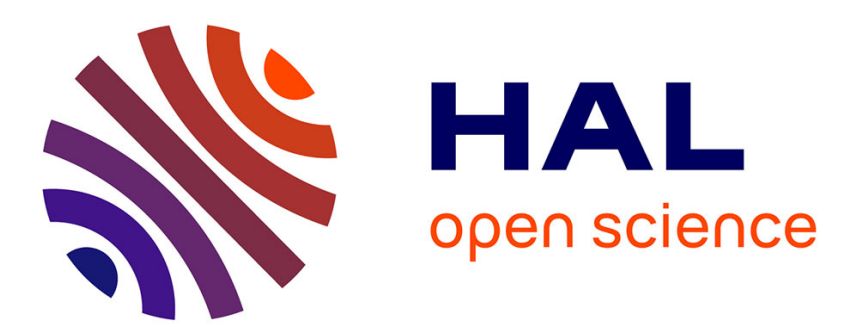

\title{
A multi-level maintenance policy for a multi-component and multi-failure mode system with two independent failure modes
}

\author{
Wenjin Zhu, Mitra Fouladirad, Christophe Bérenguer
}

\section{To cite this version:}

Wenjin Zhu, Mitra Fouladirad, Christophe Bérenguer. A multi-level maintenance policy for a multicomponent and multi-failure mode system with two independent failure modes. Reliability Engineering and System Safety, 2016, 153, pp.50-63. 10.1016/j.ress.2016.03.020 . hal-01397340

\author{
HAL Id: hal-01397340 \\ https://hal.science/hal-01397340
}

Submitted on 20 Nov 2016

HAL is a multi-disciplinary open access archive for the deposit and dissemination of scientific research documents, whether they are published or not. The documents may come from teaching and research institutions in France or abroad, or from public or private research centers.
L'archive ouverte pluridisciplinaire $\mathbf{H A L}$, est destinée au dépôt et à la diffusion de documents scientifiques de niveau recherche, publiés ou non, émanant des établissements d'enseignement et de recherche français ou étrangers, des laboratoires publics ou privés. 


\title{
A multi-level maintenance policy for a multi-component and multi-failure mode system with two independent failure modes
}

\author{
Wenjin Zhu ${ }^{\mathrm{a}, \mathrm{b}}$, Mitra Fouladirad ${ }^{1}$, Christophe Bérenguer ${ }^{\mathrm{d}}$ \\ ${ }^{a}$ Troyes University of Technology, Troyes, 10000, France \\ wenjin.zhu1017@gmail.com \\ ${ }^{b}$ Ecole des Mines de Nantes, Nantes, 44300, France \\ wenjin.zhu1017@gmail.com \\ ${ }^{c}$ Troyes University of Technology, Troyes, 10000, France \\ mitra.fouladirad@utt.fr \\ ${ }^{d}$ Univ. Grenoble Alpes, GIPSA-lab, F-38000 Grenoble, France \\ CNRS, GIPSA-lab, F-38000 Grenoble, France \\ christophe.berenguer@grenoble-inp.fr
}

\begin{abstract}
This paper studies the maintenance modelling of a multi-component system with two independent failure modes with imperfect prediction signal in the context of a system of systems. Each individual system consists of multiple series components and the failure modes of all the components are divided into two classes due to their consequences: hard failure and soft failure, where the former causes system failure while the later results in inferior performance (production reduction) of system. Besides, the system is monitored and can be alerted by imperfect prediction signal before hard failure.

Based on an illustration example of offshore wind farm, in this paper three maintenance strategies are considered: periodic routine, reactive and opportunistic maintenance. The periodic routine maintenance is scheduled at fixed period for each individual system in the perspective of system of systems. Between two successive routine maintenances, the reactive maintenance is instructed by the imperfect prediction signal according to two criterion proposed in this study for the system components. Due to the high setup cost and practical restraints of implementing maintenance activities, both routine and reactive maintenance can create the opportunities of maintenance for the other components of an individual system. The life cycle of the system and the cost of the proposed maintenance policies are analytically derived. Restrained by the complexity from both the system failure modelling and maintenance strategies, the performances and application scope of the proposed maintenance model are evaluated by numerical simulations.
\end{abstract}

Keywords: multi-component system, multiple failure modes, prediction signal, opportunistic maintenance, routine maintenance

\section{Notation}

$C_{h i} \quad i$ th hard component

$C_{s i} \quad i$ th soft component

$\left(\alpha_{i}, \lambda_{i}\right) \quad$ shape parameter and scale parameter of Weibull distribution of component $i$

$\delta$ precision, describing the proximity of the signal to the time of the actual failure 
$D_{l i}$

$A_{l i}$

$s_{i}$

$\xi_{i}(t)$

$f_{i}(\cdot)$

$f_{S i}\left(\cdot \mid t_{i}\right)$

$f_{i}^{t_{f}}\left(\cdot \mid s_{i}\right)$

$t_{f i}$

$R_{i}(\cdot \mid t)$

$H_{\text {subs. }}$

$Y_{i}$

$J_{i}(t)=\left\lceil\frac{t}{\tau}\right\rceil$

$T_{k}=\sum_{j=1}^{k} Y_{k}$

$A_{i, j}$

$a_{i, n_{i}}=\sum_{j=1}^{n_{i}} A_{i, j}$

$N_{i}(t)=\sum_{j=1}^{\infty} \mathbb{1}_{\left\{a_{i, j} \leq t\right\}}$

$A_{i, j}^{P}$

$A_{i, j}^{C}$

$A_{i, j}^{O}$

$C_{i m}^{h}$

$B_{i, j}$

$N^{S}(t)=\sum_{j=0}^{\infty} \mathbb{1}_{\left\{T_{j}<t\right\}}$

$N^{\tau}(t)=\sum_{j=0}^{\infty} \mathbb{1}_{\{j \tau<t\}}$

$b_{i, k}=\sum_{j=1}^{k} B_{i, j}$

$K_{i}=\left\lceil\frac{Y_{i}}{\tau}\right\rceil$

$N_{i}^{t}$

$C_{i m}^{s}$ accuracy, the probability of not detecting potential failure before actual failure

evaluate the closeness between the abnormal signal and the next routine maintenance

time interval of routine maintenance

expected cost for reactive maintenance without delay

expected cost for reactive maintenance with delay

age threshold, deciding the functional component that can take the opportunity to be maintained

component life of hard component $i$

design life of component $i$

age of component $i$

moment of the abnormal signal of component $i$

age of component $i$ at time $t$

Weibull pdf

conditional pdf of abnormal signal of $C_{h i}$ given the actual failure may occur at $t_{i}$ conditional pdf of life given abnormal signal occurs at $s_{i}$

lifetime of component $C_{\cdot i}$

survival function of component $C_{{ }_{i}}$ given it functions at time $t$

subsystem consists of the other components exclusive the mentioned component

$i$ th replacement cycle of the hard components

maximum number of routine maintenance actions experienced by hard component $C_{h i}$ during time interval $t$

the time of the $k$ th maintenance of the system

the $j$ th replacement cycle of component $C_{h i}$

the time of the $n_{i}$ th replacement of component $C_{h i}$

renewal function

for component $C_{. i}$, its $j$ th maintenance is preventive maintenance

for component $C_{. i}$, its $j$ th maintenance is corrective maintenance

for component $C_{.}$, its $j$ th maintenance is opportunistic maintenance

total cost caused by hard components during time interval $t$

$j$ th replacement cycle of soft component $C_{s i}$

counting process of the reactive maintenance times

counting process of the routine maintenance times

time of $k$ th maintenance of soft component $C_{s i}$

maximum number of routine maintenances experienced by soft component $C_{s i} \operatorname{during} Y_{i}$

number of soft failures during $T_{i}$

total cost caused by soft component during time interval $t$

\section{Introduction}

In the past several decades, failure models were discussed and studied concentrating on the single component system and single failure mode. However in recent years, due to the complexity of system configurations and the diversity of failure modes of the practical industrial systems, such as wind turbines and aircrafts, multi-component system modelling 
with multiple failure modes is drawing increasing attention even though it leads to additional difficulties. On the one hand, interactions (such as economic dependency, stochastic dependency, structural dependency) between components complicate the failure modelling and scheduling of maintenance. On the other hand, the complexity and effectiveness of optimizing procedure including formulations and simulations for such system are extensively more difficult than these of the single component system. Although many maintenance models have been proposed for single-component systems, they cannot be applied directly on multi-component system due to the economic dependency among the components, see in $[1,2]$. Besides, different failure modes and their effects on the system increase the modelling complexity of system performance as well as the maintenance scheduling. Moreover, it is interesting to notice that condition monitoring information has been gradually taken into account maintenance modelling. For the system whose failure mechanisms are considerably complex, the availability level of condition monitoring information decides which maintenance strategy could be established. In an offshore wind farm, where the wind turbines operate in harsh and unstable environment with low accessibility, the benefits of reliable maintenance strategy with limited available information are worthy to be studied. The introduction section firstly depicts the existing achievement of maintenance models for multi-component systems; secondly, it emphasizes some literature concentrating on the different failure modes with their definition; thirdly, it draws forth the information using for failure modelling and decision making in the existing literature. Then based on the background of the offshore wind farm, we will introduce the practical and theoretical significance of the proposed model.

In [3] the authors presented an overview of the three main groups of maintenance models for multi-component system in the existing literature: the block replacement models, group maintenance models, and opportunistic maintenance; meanwhile they emphasized the potential shortcoming of applying the regular maintenance policies directly on multi-component system, such as the low availability. To avoid these shortcomings, some researchers took maximization availability as decision criteria to optimize the decision parameters. In [4] a multi-component system is maintained by imperfect repair and perfect maintenance for each component. The periodic preventive maintenance intervals are optimized among the components according to the availability criteria by dynamic programming. In [5] the maintenance cost is dependent on the degradation state and the optimal preventive maintenance interval yielding minimal downtime is solved by integer programming. Some other researchers focused on proposing some practical maintenance strategies to maintain the availability at a given threshold. In [6] the authors considered a two-unit series system which can share the setup cost when they both need inspection or replacement. Then to coordinate inspection/replacement the opportunistic maintenance chance and inspection are scheduled separately firstly and then compromised altogether according to a given criteria. In [7] the authors considered a multi-component series system where each component is scheduled to be maintained according to its own period and the opportunistic maintenance is informed according to the expected cost. In [8] the authors studied the opportunistic maintenance of the system which is composed of multiple nonidentical and life-limited components with both economic and structural dependencies and they extended the optimization rule to improve the efficiency of decisionmaking. In [9] the authors studied a multi-component system with two maintenance actions: imperfect maintenance and replacement, by minimizing total cost and maximizing overall system reliability by metaheuristic solution methods and generational genetic algorithm. In [10] the authors studied the maintenance policies for a multi-component system with failure interactions and scheduled opportunistic maintenance by decomposing the system into mutually influential single-component systems. In [11] the role of opportunistic maintenance for multi-component system is specially pointed out. In [12] the authors studied a multi-component system with economic dependencies by solving a NP-complete dynamic grouping strategy. In [13] the authors considered the system-level and component-level periodic inspectionbased maintenance polices and solved the optimization problem for the component-level policy by the simulation based 
optimization approach with stochastic approximation. In [14] the authors emphasized the expensive maintenance setup cost and optimized the periodic maintenance by two levels: the component and the overall system according to the independency assumption with the aim of reducing the setup cost.

For a multi-component system, several failure modes can simultaneously affect on the system. Lin et.al introduced the concept of two categories of failure modes: maintainable failure and non-maintainable failure in [15]. They assumed that preventive maintenance $(\mathrm{PM})$ can reduce the hazard rate of the maintainable failure modes but cannot change the hazard rate of the non-maintainable failure modes. The mentioned PM is more inclined to the partial maintenance which can not change the inherent design and wearing of the machine. In [16] the authors extended the maintainable and non-maintainable failures by the dependent competing failure modes: the failure rate of the maintainable failure depends on the failure rate of the non-maintainable failure. Based on the similar failure models, Zhang and Wang studied a repairreplacement policy $N$, under which the system is replaced whenever the number of repairable failures reaches $N$ or the irreparable failure occurs, whichever occurs first, and they derived the explicit expression of the average cost rate in [17]. Castro [18] assumed that two failure modes are dependent: the occurrence of maintainable failures depends on the total number of non-maintainable failures from the installation of the new system. Two maintenance actions are considered: 1)the minimal repair for both failure modes and 2)the PM which only reduces the hazard rate of the maintainable failure. In [19] Wang et al. studied the maintenance strategy of a multi-component system with multiple independent failure modes. Ding and Tian studied three preventive maintenance actions on the multi-component wind turbine corresponding to the failed components and operational components considering economic dependencies and besides the opportunistic maintenance is decided by different age thresholds in [20]. In [21] the dependent and stochastic degradation of multiple components is modelled by copula methods and the influences of dependency on the optimal maintenance decision are investigated.

The condition monitoring techniques are broadly investigated in many existing literature. Jardine et al. summarized the research and developments in diagnostics and prognostic of system failure from data acquisition to data processing and their application on maintenance decision support in [22]. In [23] a framework for selecting appropriate prognostic models to predict the remaining useful life of engineering assets is studied. Although many methods have been employed (reviewed and compared in [24]), such as the time series method (ARMA)in [25], neural network modeling in [26] and piecewise deterministic Markov process in [27], the uncertainties in prognostic and prediction models are inevitable. In order to study the benefits of condition monitoring signal without losing generality and practical signification, some researchers prefer to impose the probability distribution of the life indicator/signal in maintenance models. In [28] and [29] the system is monitored and the prediction of the potential failure is related to an abnormal signal which occurs prior to the actual failure with a non-negative probability. In [30] the authors used the signals which are released by the system when it deteriorates to determine the system life time and hence plan opportunistic maintenance. In [31] the authors presented a dynamic predictive maintenance policy for a multi-component system by updating the degradation information and remaining useful life of each component and provided a comparative study.

In this paper we consider a multi-component system with two independent failure modes, which is similar as the concept proposed in [32] and [33]: soft failure and hard failure. The former usually causes system production reduction or inferior performance while the system keeps functioning; the later can cause system failure. These failure modes have important significance in engineering practice. Figure 1 provides an illustration example of the system structure. The system of systems contains $k$ identical individual systems and each system consists of $m_{1}+m_{2}$ series components (abbreviated as Comp in Figure 1), where the failure modes (abbreviated as $F M$ in Figure 1) of the first $m_{1}$ components are corresponding to hard failure and the remaining $m_{2}$ components' failures are soft failure. Certain special maintenance actions are planned 

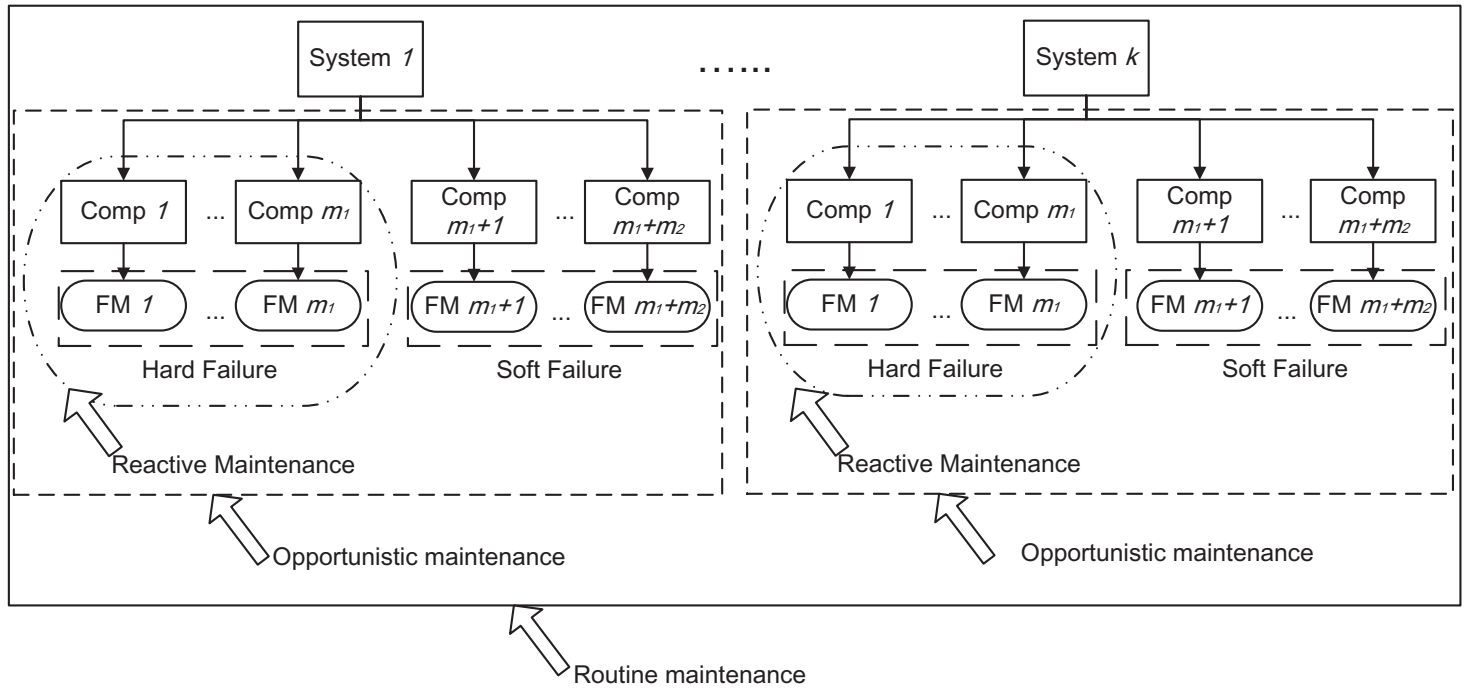

Figure 1: Schematic of the system structure and failure modes

for each system in the perspective of the system of systems. We refer them as "routine maintenance" in the rest of the paper. The components which cause hard failure are monitored and they will release some random prediction signals of a potential failure. The signals may trigger reactive maintenance for each component according to its decision threshold with respect to the framework of routine maintenance. Besides, executing each routine and reactive maintenance action requires system shutdown and hence can create the maintenance opportunities for the other components of the same system. Thus, routine, opportunistic and reactive maintenance, which are corresponding to the different levels of system structure, are referred as three levels of maintenance actions.

We take the wind turbine and wind farm as an illustrating example. An offshore wind farm usually consists of a block of wind turbines. The wind farm can be considered as the "system of systems" and each wind turbine as an individual system. The wind turbines are initially independent and identically configured. Each individual wind turbine operates separately according to its health state and environment (such as wind speed, direction and wave height). According to the failure frequency and maintenance difficulty revealed in [34-36], a wind turbine consists of the critical components such as rotor blade, hub and pitch system, main shaft, gearbox, generator, converter and tower. The failure of the gearbox, the rotor blade, the converter and the generator (which are referred as "hard failure" in this study) of the wind turbine may make the whole turbine stop working as soon as they occur. Meanwhile the failure of the control (pitch) system (which is referred as "soft failure" in this study) of the wind turbine makes it fail to adjust the blade to the right direction and the turbine is unable to make full use of the wind and results in the production loss, but the turbine can still work with such failure. In the following contents we focus on a single wind turbine in details, then plan its maintenance strategy subjecting to the co-ordinate arrangements of the wind farm which it belongs.

Due to the low accessibility of the offshore environment and the expensive setup cost, the maintenance actions, such as updating of software, lubrication and cleaning are common and periodic for all the wind turbines in the same wind farm. As the remote and built-in monitor technique and devices of wind turbine are available in wind energy practice, we assume that the system is monitored, which means that the prediction signal of potential failure is available. We adopt a general assumption that after the occurrence of the prediction signal, the actual failure time of the component is subject to a random distribution. This assumption includes the uncertainties of both the monitoring technique and the methods of signal processing. Based on the failure model the performance of the maintenance policy consists of three levels, the 
periodic routine, reactive and opportunistic maintenance, is studied comprehensively in this paper.

Our study is different from the previous studies of maintenance modeling of complex systems in that

- From the modelling's point of view, we model the system failure and maintenance strategies in a framework of "system of systems" and make it accord with the overall planning of the system of systems, which is of great importance yet has been rarely studied or even mentioned in the existing literature.

- From the available information's point of view, decision making with uncertainties is studied. The effect of uncertainties of the prediction signal on the selection of decision criterion and performance of maintenance strategies are investigated.

- From the maintenance strategies' point of view, we propose and regulate the levels of different maintenance actions according to the system structure, which makes the maintenance planning more structured.

- The mathematical expressions of the system behaviors with maintenance actions are analytically derived, which could provide some fundamental results for the further computational and numerical solutions.

The rest of this paper is organized as follows: in Section 2, we describe the details of the components' life distributions, the failure modes and the prediction signal. Based on the system description, three maintenance policies are introduced step by step in Section 3. Then in Section 4 the cost function of the maintenance policies is analytically derived. The performance of the maintenance policies are studied and analyzed in Section 5. This paper ends with concluding remarks in Section 6.

\section{System description}

This section develops further the modeling assumptions for the considered system, presented in Figure 1.

\subsection{Failure model with independent failure modes}

There are $m_{1}$ hard components and $m_{2}$ soft components in an individual system. The components constitute a set $U=\left\{1, \ldots, m_{1}, m_{1}+1, \ldots, m_{1}+m_{2}\right\}$ where the integers are corresponding to the hard components $C_{h 1}, \ldots, C_{h m_{1}}$ and soft components $C_{s 1}, \ldots, C_{s m_{2}}$. The life of component $i \in U$ is subject to Weibull distribution as follows:

$$
f_{i}(t)=\left\{\begin{array}{lr}
\alpha_{i} \lambda_{i}^{\alpha_{i}} t^{\alpha_{i}-1} e^{-\left(\lambda_{i} t\right)^{\alpha_{i}}} & \text { if } t \neq 0 \\
0 & \text { otherwise }
\end{array}\right.
$$

where $\lambda_{i}$ is the scale parameter and $\alpha_{i}$ is the shape parameter. The mean time to failure is $\operatorname{MTT} F_{i}=\frac{1}{\lambda_{i}} \Gamma\left(\frac{1}{\alpha_{i}}+1\right)$. The failure rate for component $i$ is

$$
z_{i}^{n}(t)=\alpha_{i} \lambda_{i}^{\alpha_{i}} t^{\alpha_{i}-1}
$$

The mean residual life of the component $i$ knowing that it functions at time $t$ is defined as follows:

$$
\begin{aligned}
M R L_{i}(t)=\mathbb{P}\left(L_{i}>t+x \mid L_{i}>t\right) & =\frac{1}{R_{i}(t)} \int_{0}^{\infty} R_{i}(x+t) d x \\
& =\frac{e^{\left(\lambda_{i} t\right)^{\alpha_{i}}}}{\alpha_{i} \lambda_{i}} \Gamma\left(\frac{1}{\alpha_{i}},\left(\lambda_{i} t\right)^{\alpha_{i}}\right)
\end{aligned}
$$

where $L_{i}$ is the lifetime of component $i$. Let us recall the following assumptions:

- The hard components form a series sub-system within a system and any hard component failure leads to a selfannouncing system failure. 

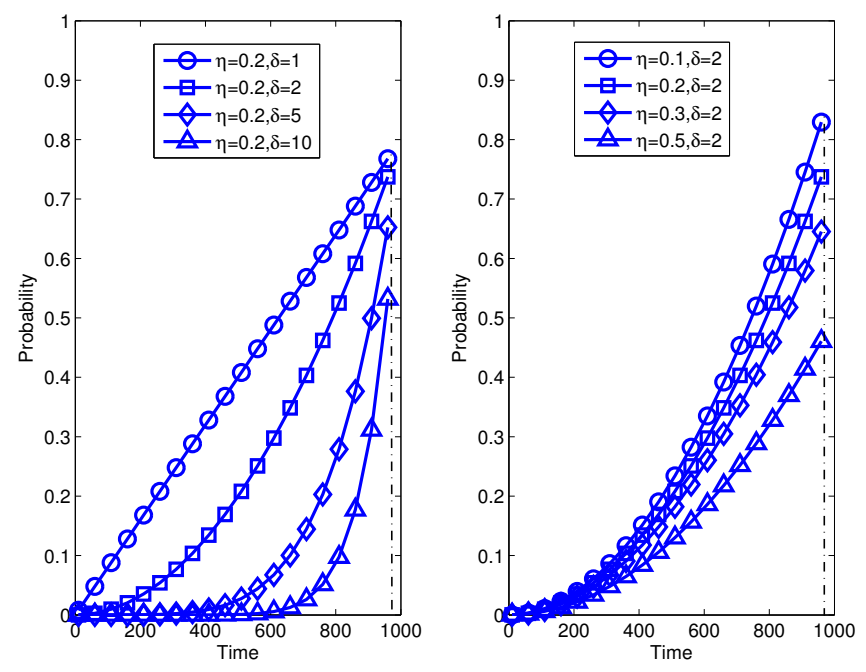

Figure 2: Sensitivity of the parameters $\delta$ and $\eta$ of conditional distribution of imperfect prediction signal given the component life be 1000

- The failure of any soft component results in a hidden soft failure. The system can work with a soft failure only undergoing the production loss during the soft failure.

- The maintenance action causes downtime cost.

\subsection{Prediction signal by monitoring}

As mentioned in [28], a monitored system may have the positive probability that an imperfect prediction signal is prior to an actual failure. Assume that for each hard-type component, an imperfect prediction signal may show up before the corresponding hard failure. The term Accuracy is designed to describe the likelihood of predicting a system failure prior to an actual failure. Precision describes the proximity of the signal to the moment of the actual failure. By adopting a similar conditional density and distribution function as in [28], we define the accuracy and precision of the failure prediction. For hard-type component $i$, let $s_{i}$ be the random time when the imperfect prediction signal happens and $t_{f i}$ be the failure time of component $i$. Let $\delta \geq 1$ be the prediction precision, $(1-\eta)$ be prediction accuracy and $0 \leq \eta \leq 1$, the conditional distribution that an imperfect prediction signal shows up given the life of the hard component $t_{f i}$ is:

$$
\begin{gathered}
\mathbb{P}\left(s_{i}<s \mid t_{f i}=x\right)=F_{s i}(s \mid x)=\left\{\begin{array}{lr}
(1-\eta)\left(\frac{s}{x}\right)^{\delta} & \text { if } 0 \leq s \leq x \\
1 & s>x
\end{array}\right. \\
f_{s i}(s \mid x)=\left\{\begin{array}{lr}
\delta(1-\eta) \frac{s^{\delta-1}}{x^{\delta}} & \text { if } 0 \leq s \leq x \\
\eta & s>x
\end{array}\right.
\end{gathered}
$$

where $\eta=0$ means that the imperfect prediction signal shows up before the actual failure with probability 1 . Figure 2 gives some examples of the time distribution when the imperfect prediction signal occurs given the component fails at time 1000 .

$\eta$ and $\delta$ could be estimated according to the historical lifetime data and we omit this procedure and technique in this paper. In the following context, we suppose that $\eta$ and $\delta$ are known. Thus the conditional survival analysis of the component which receives the prediction signal is actually a Bayesian model. Let $R_{i}\left(t-s_{i} \mid s_{i}\right)$ be the survival function conditional on the imperfect prediction signal of component $i$ occurs at $s_{i}$ for $t>s_{i}$ :

$$
R_{i}\left(t-s_{i} \mid s_{i}\right)=\mathbb{P}\left(t_{f i}>t \mid t_{f i}>s_{i}\right) .
$$


Given the imperfect prediction signal occurs at $s_{i}$, according to Bayesian theory, the conditional survival pdf is

$$
f_{i}^{t_{f}}\left(t \mid s_{i}\right)=\frac{f_{S i}\left(s_{i} \mid t\right) f_{i}(t)}{\int_{s_{i}}^{\infty} f_{S i}\left(s_{i} \mid t\right) f_{i}(t) d t}
$$

For the denominator, after simplification, we have

$$
\begin{aligned}
\int_{s_{i}}^{\infty} f_{S}\left(s_{i} \mid t\right) f_{i}(t) d t & =\int_{s_{i}}^{\infty} \frac{\delta}{t}\left(\frac{s_{i}}{t}\right)^{\delta-1} \alpha_{i} \lambda_{i}^{\alpha_{i}} t^{\left(\alpha_{i}-1\right)} e^{-\left(\lambda_{i} t\right)^{\alpha_{i}}} d t \\
& =\delta s_{i}^{\delta-1} \lambda_{i}^{\delta} \Gamma\left(1-\frac{\delta}{\alpha_{i}},\left(\lambda_{i} s_{i}\right)^{\alpha_{i}}\right)
\end{aligned}
$$

Hence the conditional survival pdf given the imperfect prediction signal occurs at $s_{i}$ is

$$
f_{i}^{t_{f}}\left(t \mid s_{i}\right)=\frac{\alpha_{i}}{t \Gamma\left(1-\frac{\delta}{\alpha_{i}},\left(\lambda_{i} s_{i}\right)^{\alpha_{i}}\right)}\left(\lambda_{i} t\right)^{\alpha_{i}-\delta} e^{-\left(\lambda_{i} t\right)^{\alpha_{i}}}
$$

The survival function of the hard component given the imperfect prediction signal occurs at $s_{i}$ is

$$
\begin{aligned}
R_{i}\left(t-s_{i} \mid s_{i}\right) & =\mathbb{P}\left(t_{f}>t \mid t_{f}>s_{i}\right)=\int_{t}^{\infty} f_{i}^{t_{f}}\left(x \mid s_{i}\right) d x \\
& =\frac{\Gamma\left(\alpha_{i} \delta+1,\left(\lambda_{i} t\right)^{\alpha_{i}}\right)}{\Gamma\left(1-\frac{\delta}{\alpha_{i}},\left(\lambda_{i} s_{i}\right)^{\alpha_{i}}\right)}
\end{aligned}
$$

\section{Maintenance policy}

\subsection{Maintenance level}

In this section we present three different maintenance schedules in details based on the system structure and failure models sketched in Fig 1: routine maintenance at the system of systems level (i.e. the wind farm level in our illustrating example) in section 3.1.1, reactive maintenance for the component level in section 3.1 .2 and opportunistic maintenance for the individual system level, (i.e the wind turbine level $\mathrm{n}$ our illustrating example) in section 3.1.3.

\subsubsection{System of systems level: Routine maintenance}

In most of the existing literature, the routine maintenance is not distinguished from the general periodic maintenance for individual component/system. For an individual system, it does not make any difference. However, for the system, such as the wind farm which consists of a group wind turbines and each wind turbine is a multi-component system, the routine maintenance is planned for the whole farm while the specific maintenance strategy is planned for the individual turbine. Actually, the routine maintenance activities, such as updating of software, lubrication and cleaning of the system, do not involve the direct maintenance of the specific component/system, but they affect their degradation or failure.

The routine maintenance is scheduled in advance in order to guarantee the availability of the staff and the equipments. Thus the cost for the preparation work of maintenance actions is considered. In this paper, such cost is referred as the "setup" cost. The period of routine maintenance is usually prescribed by the instructions of the manufacturer and thus its selection range is limited. Assume that the routine maintenance is scheduled at fixed time intervals equal to $\tau$. As the soft failure is hidden and causes the production loss, the soft components should be inspected during each routine maintenance in order to reduce the production loss.

Furthermore, since it is possible that the imperfect prediction signal does not occur before an actual hard failure, the preventive maintenance of hard component at the routine maintenance can reduce the risk of hard failure. 


\subsubsection{Component level: Reactive maintenance}

An imperfect prediction signal may show up before a hard failure occurs. On the one hand, the setup cost of a reactive maintenance between two routine maintenances is relatively more expensive than that of the routine maintenance; on the other hand, there is a time delay between the imperfect prediction signal and the actual failure. People may consider the idea that each imperfect prediction signal can trigger a reactive maintenance, however it may cause some prematurely preventive maintenances. Hence the way that the signal is treated is very important. We consider the following criteria:

- (Tolerance criteria: Save the setup cost) If an imperfect prediction signal shows up and it is close to the next routine maintenance time, delaying the reactive maintenance until the routine maintenance could be a cost-efficient solution. On the contrary, if the imperfect prediction signal shows up quite early, it is risky to delay the reactive maintenance. Thus a designed parameter, $\varepsilon$, will be introduced to evaluate the closeness $v_{i}$ (which is calculated by $\left.v_{i}=\left[s_{i}-(n-1) \tau\right] / \tau\right)$ between the imperfect prediction signal of component $C_{h i}$ and the next routine maintenance. In [20] a similar parameter has been used to group the components whose inspection intervals are close enough. Let the tolerance $\varepsilon$ vary from 0 to 1 , where $\varepsilon=1$ means the reactive maintenance is suggested to be delayed at the next routine maintenance and $\varepsilon=0$ means that the reactive maintenance will be executed immediately. For component $C_{h i}$

- if $v_{i}>\varepsilon$, then delay the reactive maintenance until the next reactive maintenance, a hard failure or the next routine maintenance, whichever occurs first;

- if $v_{i}<\varepsilon$, then the corresponding component is maintained immediately.

- (Expected cost criteria: Reduce the risk of hard failure) Concerning the dependence between the prediction signal and the age of the hard component, we should consider the residual life of the component whose prediction signal shows up. Let $E C_{i m}^{i}$ and $E C_{w d}^{i}$ be the expected cost of the reactive maintenance of hard component $C_{h i}$ without delay and with delay respectively. $E C_{i m}^{i}$ and $E C_{w d}^{i}$ will be calculated online and be compared with each other. After the comparison,

- if $E C_{i m}^{i}>E C_{w d}^{i}$, then delay the reactive maintenance until the next reactive maintenance, a hard failure or the next routine maintenance, whichever occurs first;

- if $E C_{i m}^{i}<E C_{w d}^{i}$, then the corresponding component is maintained immediately.

\subsubsection{System level: Opportunistic maintenance}

During a maintenance action, the system is shut down and a downtime cost is incurred. If the other components of the same system can take the opportunity to be preventively maintained, the downtime cost and possible set up cost can be shared by several components. Thus at the beginning of a maintenance, either corrective or preventive, when the component corresponding to the actual or potential failure is identified, some of the other components of the corresponding system will be opportunistically maintained. Inspections are required to identify the hidden soft failure. The age could be an alternative measurement of the health state of the component when deciding the opportunistic maintenance. The age threshold parameter $q_{1}$ is introduced to decide which component should be maintained opportunistically. A high value of $q_{1}$ leads to less opportunities for the functional components to be maintained.

\subsection{Maintenance strategy integrating the three maintenance actions}

For component $i$, let $t_{f i}$ denote the component life, $D_{l i}$ denote the design life of the component, $\xi_{i}$ denote the age of component and $s_{i}$ denote the moment of the imperfect signal. Maintenance procedure is performed as follows: 
1. Reactive maintenance according to closeness criteria (component level: Save the setup cost)

- If the first prediction signal is released by $C_{h i}$ and occurs at $s_{i}$ between the $(n-1)$ th and $n$th routine maintenances, record the time and calculate the closeness $v_{i}$ by $v_{i}=\left[s_{i}-(n-1) \tau\right] / \tau$.

- If $v_{i}<\varepsilon$, a reactive maintenance is scheduled immediately and the other components of the same turbine may take the opportunity to be maintained.

- If $1>v_{i}>\varepsilon$, delay the reactive maintenance corresponding to component $i$ and all the other components whose prediction signals satisfying $1>v_{j}>v_{i}$ until $n \tau, j \neq i, j \in U$.

- If a hard failure occurs during the delay at $t_{f i}$, then the reactive maintenance will be advanced at $t_{f i}$. All the components whose prediction signals occurred between $s_{i}$ and $t_{f i}$ are maintained.

2. Reactive maintenance according to expected cost criteria (component level: Reduce the risk of hard failure)

- If a prediction signal occurs at $s_{i}$ between two successive routine maintenances, record the time and calculate the expected cost $E C_{i m}^{i}$ and $E C_{w d}^{i}$.

- If $E C_{i m}^{i}<E C_{w d}^{i}$, a reactive maintenance is to be scheduled immediately and the other components of the same turbine may take the opportunity to be maintained.

- If $E C_{i m}^{i}>E C_{w d}^{i}$, the reactive maintenance corresponding to component $i$ and all the other components with prediction signals occurred and satisfying $E C_{i m}^{i}>E C_{w d}^{i}$ is postponed until $n \tau$.

- If a hard failure occurs during the delay at $t_{f j}$ or a component $C_{h k}$ satisfying $E C_{i m}^{k}<E C_{w d}^{k}$ at $s_{k}$, then the reactive maintenance will be advanced at $t_{f j}$ or $s_{k}, k \neq i$. All the components whose prediction signal occurred between $s_{i}$ and $t_{f j}$ or $s_{k}$ are maintained.

3. Opportunistic maintenance according to age threshold (system level: $q_{1}, q_{2}$ and $q_{3}$ )

- If a hard component needs to be replaced, either preventively or correctively, all the other components of the same turbine may take the opportunity to be maintained.

- For a soft-type component $C_{s i}$, if it is not failed and its age $\xi_{l i}$ does not exceed the age threshold $q_{1} D_{l i}$ at the reactive maintenance time or $q_{3} D_{l i}$ at the routine maintenance time, no maintenance action is carried out on it.

- If $C_{s i}$ is not failed and its age exceeds $q_{1} D_{l i}$ at the reactive maintenance time or $q_{3} D_{l i}$ at the routine maintenance time, it is opportunistically and preventively replaced.

- For a hard-type component $C_{h j}$, if it is not failed, it does not show prediction signal and its age $\xi_{l j}$ is less than $q_{1} D_{l j}$ at the reactive maintenance time or $q_{2} D_{l j}$ at the routine maintenance time, no maintenance action is taken on it.

- If $C_{h j}$ shows prediction signal or its age exceeds $q_{1} D_{l j}$ at the reactive maintenance time or $q_{2} D_{l j}$ at the routine maintenance time, it is opportunistically and preventively replaced.

4. Routine maintenance (system of systems' level: $\tau$ )

- At scheduled times $n \tau, n \in N$, if there are delayed reactive and opportunistic maintenances, execute them according to their own rules. 


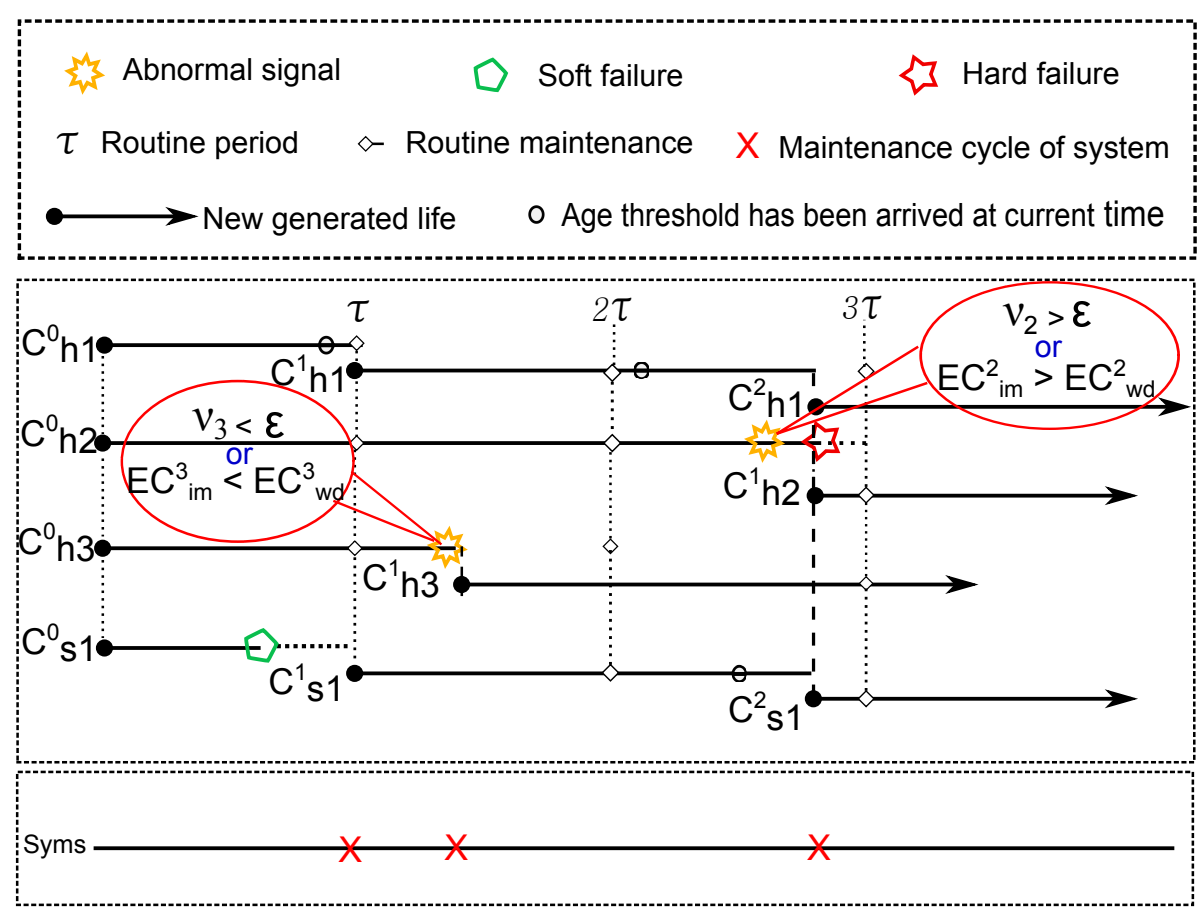

Figure 3: The detailed procedure of how the tolerance criteria or expected cost criteria conducts the maintenance action initiated by prediction signal

- If no delayed maintenance, inspect the state of soft components and make maintenance decision on them. At the same time, check the possibility of opportunistic maintenance.

Take Figure 3 as an example, the system consists of three hard components and a soft component. During the first routine period, the age of $C_{h 1}$ attains its threshold and $C_{s 1}$ fails. Both $C_{h 1}$ and $C_{s 1}$ are maintained at time $\tau$. During the second routine period $(\tau, 2 \tau], C_{h 3}$ releases a prediction signal. According to tolerance criteria or expected cost criteria, the reactive maintenance is suggested to be carried out without delay. During the $(2 \tau, 3 \tau], C_{h 2}$ releases a prediction signal. According to tolerance criteria or expected cost criteria, the reactive maintenance is suggested to be delayed. However, $C_{h 2}$ fails before $3 \tau$. Thus the reactive maintenance is advanced immediately. Meanwhile $C_{h 1}$ and $C_{s 1}$ satisfy the condition of opportunistic maintenance. As a result, $C_{h 1}, C_{h 2}$ and $C_{s 1}$ are maintained jointly.

\section{Cost analysis of the maintenance policy}

\subsection{Cost definition}

The following cost items are included in this study for the evaluation of the average long run cost per time unit:

- $C_{s t}$ : setup cost of an individual system for reactive maintenance;

- $C_{t}$ : setup cost of a system for routine maintenance and $C_{s t}>C_{t}$;

- $C_{s p}(i)\left(\right.$ respectively $\left.C_{h p}(i)\right)$ : preventive replacement cost of soft component $C_{s i}$ (respectively hard component $\left.C_{h i}\right)$;

- $C_{s f}(i)\left(\right.$ respectively $\left.C_{h f}(i)\right)$ : corrective replacement cost of soft component $C_{s i}$ (respectively hard component $C_{h i}$ );

- $C_{s u}\left(\right.$ respectively $\left.C_{h u}\right)$ : downtime cost of soft component $C_{s i}$ (respectively hard component $C_{h i}$ );

- $C_{i s}(i)$ : inspection cost for soft components $C_{s i}$;

- $C_{l}$ : unit production loss of soft failure. 


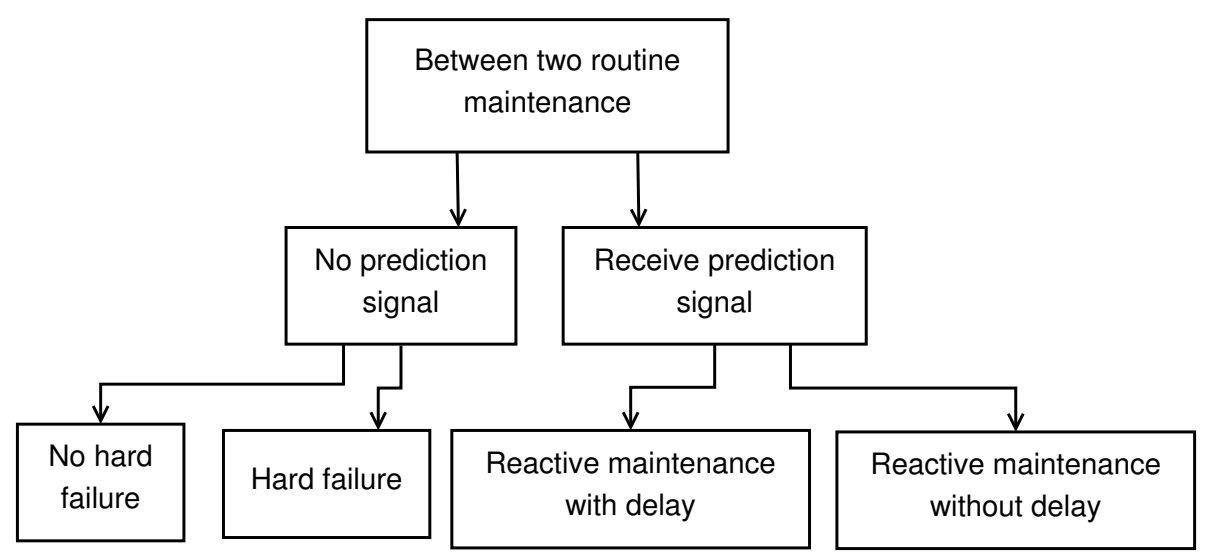

Figure 4: The possible scenarios of the proposed maintenance policy between two routine maintenances. Superscript $i$ of $C_{\cdot{ }_{j}}$ denotes the $i$ th maintenance of component $C_{\cdot j} . \quad v_{j}$ is the closeness of $C_{h j} ; \varepsilon$ is the tolerance threshold. $E C_{i m}^{j}$ and $E C_{w d}^{j}$ are the expected cost of reactive maintenance of component $C_{h j}$ without and with delay respectively.

\subsection{Cost analysis and formulation}

Between two successive routine maintenances, the following scenarios are possible(see also Figure 4):

1. Neither hard failure nor imperfect prediction signal occurs.

2. Hard failure occurs without warning any imperfect prediction signal.

3. An imperfect prediction signal $s_{i}$ corresponding to $C_{h i}$ shows up and the reactive maintenance needs to be executed without delay. Check the possibility of opportunistic maintenance.

4. An imperfect prediction signal $s_{i}$ corresponding to $C_{h i}$ shows up and the reactive maintenance can be delayed; the maintenance of all the other components whose imperfect prediction signals show up after $s_{i}$ and satisfy the delay conditions can be delayed until the conditions for an immediate reactive maintenance satisfy. Check the possibility of opportunistic maintenance.

5. After the reactive maintenance, wait for the next imperfect prediction signal, the hard failure or the next routine maintenance, whichever occurs first.

\subsubsection{Average unit cost}

The total cost induced during time $t$ is composed of the total maintenance cost of the soft and hard components respectively. Let $C_{i m}^{h}(t)$ and $C_{i m}^{s}(t)$ be the total maintenance cost caused by the hard and soft components respectively from time 0 to $t$. The average unit cost during $t$ can be calculated as follows

$$
\mathbb{E} C(t)=\mathbb{E}\left(C_{i m}^{h}(t)\right)+\mathbb{E}\left(C_{i m}^{s}(t)\right)
$$

where

$$
C_{i m}^{h}(t)=\sum_{i=1}^{m_{1}}\left(N^{p, i}(t)+N^{o, i}(t)\right) C_{h p}(i)+N^{c, i}(t)\left(C_{h f}(i)+C_{s t}\right)+C_{t}+C_{h u}
$$

where $N^{p, i}(t), N^{o, i}(t)$ and $N^{c, i}(t)$ are respectively the number of preventive, opportunistic and corrective maintenance of the hard component $i$ before time $t$. Moreover,

$$
C_{i m}^{s}(t)=\sum_{m_{1}+1=m_{2}}^{n} N_{i}^{f}(t)\left(C_{s f}+C_{l}\right)+N_{i}^{p}(t) C_{s p}+N_{i}^{i}(t) C_{i s}
$$

where $N_{i}^{f}$ and $N_{i}^{p}, N_{i}^{i}$ are respectively the number of the failures, preventive maintenance and inspections of the soft component $i$ before $t$. All these quantities are mathematically defined and calculated in section Appendix A and Appendix B. 


\subsection{The expected cost criteria of the reactive maintenance}

The reactive maintenance is carried out when the cost criteria given in section 3.1.2 is satisfied. The criteria is based on $E C_{i m}^{i}$ and $E C_{w d}^{i}$ : the expected cost of the reactive maintenance of hard component $C_{h i}$ without delay and with delay respectively. These costs can be calculated as follows. Assume the prediction signal corresponding to the hard component $C_{h i}$ show up during the $n$th cycle(i.e, between $(n-1)$ th and $n$th routine maintenance). Let $\xi_{i}(t)$ denote the age of $C_{h i}$ at last maintenance of the system at time $t$. When the prediction signal occurs at $s_{i}$, the cost for the delayed and immediate reactive maintenance are as follows:

$$
\begin{aligned}
E C_{w d}^{i}(t)= & {\left[1-R_{i}\left(t-s_{i} \mid s_{i}\right)\right]\left(C_{h f}(i)+C_{s t}\right)+R_{i}\left(t-s_{i} \mid s_{i}\right)\left(C_{h p}(i)+C_{t}\right)+} \\
& C_{h u}+C_{l} \cdot \max \left\{0,\left[t-\min _{j \in M_{2}}\left(M R L_{j}\left(\xi_{j}\left(s_{j}\right)\right)+\xi_{j}\left(s_{j}\right)\right)\right]\right\}
\end{aligned}
$$

where $M_{2}$ is the subset of soft components, the mean residual life $M R L_{j}$ and the residual life $R_{i}$ are defined by the equation (3). Moreover,

$$
E C_{i m}^{i}=C_{h p}(i)+C_{h u}+C_{s t}+C_{l} \max \left\{0,\left[s_{i}-\min _{j \in M_{2}}\left(M R L_{j}\left(\xi_{j}\left(s_{j}\right)\right)+\xi_{j}\left(s_{j}\right)\right)\right]\right\}
$$

\section{Numerical simulations and results analysis}

\subsection{Average unit cost by simulation}

The maintenance policy based on the proposed complex system can result in many random scenarios. Although we mathematically derive some parts of the cost calculation, it is still difficult to solve the optimization problem numerically even for a low number of components. The Monte Carlo simulations method is an alternative method to simulate complex random events. Let the time period of the simulation be $N \tau$, where $N$ is a positive integer. The minimization of the following cost criteria will be used to optimize the parameters $\left(\tau, \varepsilon, q_{1}, q_{2}, q_{3}\right)$ for the tolerance criteria policy and $(\tau$, $\left.q_{1}, q_{2}, q_{3}\right)$ for the expected cost criteria policy:

$$
E\left(C_{\infty}\right)=\lim _{t \rightarrow \infty} \frac{C(t)}{t}=\lim _{t \rightarrow \infty} \frac{C_{i m}^{h}(t)+C_{i m}^{s}(t)}{t},
$$

The main programme is based on the Algorithm 1. Here we choose a special case that $\eta=0$, i.e., the prediction signal occurs always before the actual hard failure with probability 1 . When $0<\eta<1$, the procedure is similar.

For the tolerance criteria which is used to instruct the reactive maintenance, the procedure of programme is similar to "Algorithm1".

\subsection{Parameter setting}

Take the mean of the Weibull distribution as the designed life for each component. In Table 1 we list the parameters of the component life distribution, the designed life of each component.

\subsection{Tolerance criteria policy}

The variables that need to be optimized are $\left(\tau, \varepsilon, q_{1}, q_{2}, q_{3}\right)$. Let us recall the meaning of these parameters:

- $\tau$ is the period of routine maintenance;

- $\varepsilon$ is the tolerance threshold, which decides the reactive maintenance is to be executed immediately after a signal or be delayed; 


\section{Algorithm 1 Procedure of the expected cost criteria during $n$th cycle \\ Require: \\ Initialization of the set $E_{a b}=\emptyset$ : the component which shows prediction signal; \\ Initialization of the set $E_{o r}=\emptyset$ : the component which takes the opportunity;}

\section{Ensure:}

The cumulated cost of $n$th cycle;

1: Record the $i$ th prediction signal $s_{n i}$ and the corresponding component $j: E_{a b}=E_{a b} \cup j$; sort $E_{a b}=\left\{1^{\prime}, 2^{\prime}, \ldots, m^{\prime}\right\}$ according to the value of $s_{n i}$ with increasing order;

2: while $E_{a b} \neq \emptyset$ do

3: $\quad$ Calculate the $E C_{d e}^{1^{\prime}}(n \tau)$ and $E C_{r e}^{1^{\prime}}(n \tau)$ for the fist component $1^{\prime} \in E_{a b}$;

4: $\quad$ if $E C_{d e}^{1^{\prime}}(n \tau)<E C_{r e}^{1^{\prime}}(n \tau)$ then

5: $\quad$ Delay the maintenance of component $1^{\prime}$ until $\min \left(n \tau, s_{n 2^{\prime}}\right)$;

6: $\quad$ if A hard failure occurs at $s_{n j} \leq t_{f k} \leq n \tau$ by component $k$ then

7: $\quad$ Update $E_{\text {or }}$ and maintain the failed component $k$ and $E_{\text {or }}$ immediately; renew the age and generate the new lifetime; update $E_{a b}=E_{a b} \backslash\left(k \cup E_{o r}\right)$;

Update the cost;

8: $\quad$ else if No hard failure occurs: $\min \left(t_{f i^{\prime}}\right) \geq n \tau$ then

9: $\quad$ Update the cost and go to the next cycle;

10: $\quad$ else

11: Update $E_{\text {or }}$ and maintain the component $1^{\prime}$ and $E_{\text {or }}$ immediately; renew the age and generate the new lifetime; update $E_{a b}=E_{a b} \backslash\left(1^{\prime} \cup E_{o r}\right)$;

Update the cost;

$$
\begin{aligned}
& \text { 12: } \quad \text { end if } \\
& \text { 13: } \quad \text { end if }
\end{aligned}
$$

14: end while

Table 1: Parameter setting

\begin{tabular}{lllllll}
\hline Item & $C_{h 1}$ & $C_{h 2}$ & $C_{h 3}$ & $C_{h 4}$ & $C_{h 5}$ & $C_{h 6}$ \\
\hline$\alpha$ & 10 & 6 & 8 & 5 & 4 & 7 \\
$\lambda$ & $4 \times 10^{-4}$ & $3 \times 10^{-4}$ & $5 \times 10^{-4}$ & $4.5 \times 10^{-4}$ & $3.5 \times 10^{-4}$ & $4 \times 10^{-4}$ \\
$D_{l \bullet}$ & $2.378 \times 10^{3}$ & $3.092 \times 10^{3}$ & $1.883 \times 10^{3}$ & $2.040 \times 10^{3}$ & $2.590 \times 10^{3}$ & $2.339 \times 10^{3}$ \\
\hline Item & $C_{h 7}$ & $C_{h 8}$ & $C_{h 9}$ & $C_{s 1}$ & $C_{s 2}$ & - \\
\hline$\alpha$ & 2 & 8 & 5 & 2 & 3 & - \\
$\lambda$ & $5 \times 10^{-4}$ & $4.5 \times 10^{-4}$ & $3 \times 10^{-4}$ & $10^{-3}$ & $2 \times 10^{-3}$ & - \\
$D_{l \bullet}$ & $1.772 \times 10^{3}$ & $2.093 \times 10^{3}$ & $3.061 \times 10^{3}$ & $8.86 \times 10^{2}$ & $4.46 \times 10^{2}$ & - \\
\hline \hline
\end{tabular}




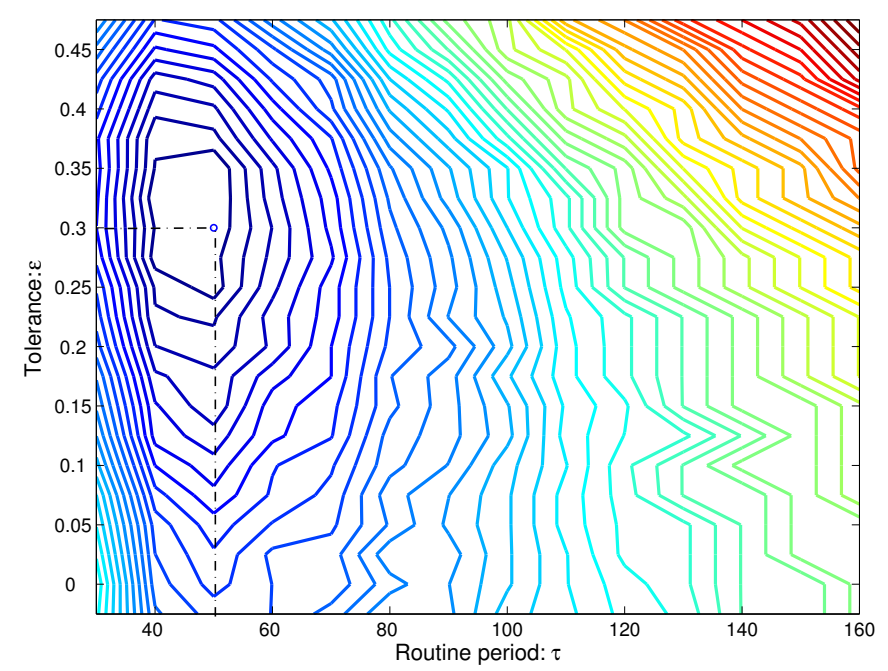

Figure 5: Tolerance criteria policy- Iso-level curves of expected average maintenance cost as a function of $(\tau, \varepsilon)$ for $q_{2}=1.3, q_{3}=1, \eta=0, \delta=4$; with 9 hard components and 2 soft components for hard component, $C \cdot f=100, C \cdot p=60 ;$ for soft component $C \cdot f=100, C \cdot p=80 ; C_{u s}=10$, $C_{u h}=50, C_{i s}=50, C_{t}=5$

- $q_{1}$ decides the age preventive/opportunistic maintenance threshold of both hard and soft component during the reactive maintenance;

- $q_{2}$ and $q_{3}$ decide the age preventive thresholds for hard and soft component respectively at routine maintenance.

We study the effect of $\delta$ (Precision, the parameter to describe the proximity of the signal to the time of the actual failure, see Fig 2) on the performance of tolerance criteria policy of the system with 9 hard components and 2 soft components. When there are a large number of hard components, it is possible to receive several prediction signals during the same period. Hence the advantage of the delay by the tolerance criteria $\varepsilon$ can be shown more clearly. Figure 5 gives an example of the contour of the optimal decision-making variables $(\tau=50, \varepsilon=0.3)$ by fixed $q_{2}$ and $q_{3}$, where the optimal $q_{1}=0.9$. For each $\tau$, the optimization stops when $N=10000$ routine maintenance times are finished.

When $\varepsilon=1$, the maintenance policy with the tolerance criteria (MPTC) degenerates as the regular opportunistic policy (ROP), i.e, when the prediction signal occurs, the reactive maintenance is executed on the component which sends out the prediction signal. Table 2 lists the numerical results of the comparison of the MPTC with $0 \leq \varepsilon<1$ and the ROP with $\varepsilon=1$ when $\delta$ varies from 1 to 10 . For the extreme case $\varepsilon=0$, all the reactive maintenances are definitely delayed until the next routine maintenance or hard failure, whichever occurs first. For $\varepsilon=1$, all the reactive maintenances are executed immediately. It can be noticed in Table 2, the performance of the MPTC which delays the reactive maintenance is more cost-effective than the ROP. As the precision of the signal $\delta$ increases, $E C_{\infty}$ decrease and becomes stable. At the same time, the optimal $\varepsilon$, i.e, $\varepsilon^{*}$ approaches to 1 and the effect of $\varepsilon$ does not highlight any more. That is because the fact that when the signal is precise enough, it is no need to take the risk to delay the reactive maintenance. As production loss $C_{l}$ increases, the cost caused by soft failure is expensive and the reactive maintenance without delay can reveal the soft failure earlier. Thus when $C_{l}$ is expensive as $C_{. f} / 10$, the optimal $\varepsilon^{*}$ is close to 1 and the MPTC is as cost-effective as the ROP.

\subsection{Expected cost criteria policy}

The decision-making variables of expected cost criteria policy are $\left(\tau, q_{1}, q_{2}, q_{3}\right)$. We start from the effect of $\delta$ for fixed $\eta=0.2$ and the simulation results are listed in Table 3 . The main results are concluded as follows: 
Table 2: Tolerance criteria: The effect of $\varepsilon$ with $\eta=0.2, C_{h f}=C_{s f}=100, C_{h p}=60, C_{s p}=80, C_{h u}=50, C_{s u}=10, C_{s t}=50, C_{t}=5$. $C_{\text {signal }}=\delta / 1000$ per time unit for each hard component, considering the maintenance policy with the tolerance criteria (MPTC) and the regular opportunistic policy $(\mathrm{ROP})$

\begin{tabular}{lcccl}
\hline$C_{l}$ & $C_{l}=0.1$ & \multicolumn{3}{c}{$C_{l}=1$} \\
\hline $\mathrm{P}$. & $M P T C$ & $R O P$ & $M P T C$ & $R O P$ \\
\hline$\delta$ & $\left(\varepsilon^{*}, E C_{\infty}\right)$ & $E C_{\infty}$ & $\left(\varepsilon^{*}, E C_{\infty}\right)$ & $E C_{\infty}$ \\
\hline 1 & $(0.00,0.958)$ & 1.180 & $(0.00,0.987)$ & 1.250 \\
2 & $(0.20,0.751)$ & 0.911 & $(0.20,0.766)$ & 0.934 \\
3 & $(0.60,0.703)$ & 0.893 & $(0.60,0.731)$ & 0.907 \\
4 & $(0.80,0.703)$ & 0.805 & $(0.80,0.731)$ & 0.897 \\
5 & $(0.80,0.700)$ & 0.761 & $(0.80,0.730)$ & 0.802 \\
10 & $(0.80,0.704)$ & 0.792 & $(0.80,0.733)$ & 0.810 \\
\hline \hline$C_{l}$ & $C_{l}=5$ & & $C_{l}=10$ & \\
\hline $\mathrm{P}$. & $M P T C$ & $R O P$ & $M P T C$ & $R O P$ \\
\hline$\delta$ & $\left(\varepsilon^{*}, E C_{\infty}\right)$ & $E C_{\infty}$ & $\left(\varepsilon^{*}, E C_{\infty}\right)$ & \\
\hline 1 & $(0.00,1.246)$ & 1.467 & $(0.00,1.317)$ & 1.694 \\
2 & $(0.00,0.902)$ & 1.142 & $(0.00,1.057)$ & 1.327 \\
3 & $(0.60,0.837)$ & 1.001 & $(0.20,0.969)$ & 1.114 \\
4 & $(0.60,0.843)$ & 0.953 & $(0.60,0.976)$ & 1.003 \\
5 & $(0.60,0.844)$ & 0.903 & $(0.60,0.980)$ & 0.997 \\
10 & $(1.00,0.846)$ & 0.846 & $(1.00,0.985)$ & 0.985 \\
\hline \hline
\end{tabular}


Table 3: Expected cost criteria: The effect of $\delta$ with $\eta=0.2, C_{. f}=100, C_{h p}=60, C_{s p}=80, C_{h u}=50, C_{s u}=10, C_{s t}=50, C_{t}=5$. $C_{\text {signal }}=\delta / 1000$ per time unit for each hard component

\begin{tabular}{lcc}
\hline$\delta \backslash C_{l}$ & $C_{l}=0.1$ & $C_{l}=1$ \\
\hline & $\left(E C_{\infty}, q_{1}, q_{2}, q_{3}\right)$ & $\left(E C_{\infty}, q_{1}, q_{2}, q_{3}\right)$ \\
\hline 1 & $(0.930,0.8,1.1,1.2)$ & $(0.955,0.8,1.2,1.0)$ \\
2 & $(0.721,0.7,1.1,1.2)$ & $(0.744,0.6,1.3,1.1)$ \\
3 & $(0.710,0.6,1.3,1.2)$ & $(0.736,0.8,1.2,1.1)$ \\
4 & $(0.717,0.8,1.2,1.2)$ & $(0.746,0.7,1.1,1.1)$ \\
5 & $(0.721,0.8,1.2,1.2)$ & $(0.753,0.6,1.3,1.1)$ \\
10 & $(0.732,0.6,1.3,1.2)$ & $(0.761,0.6,1.3,1.1)$ \\
\hline$\delta \backslash C_{l}$ & $C_{l}=5$ & $C_{l}=10$ \\
\hline & $\left(E C_{\infty}, q_{1}, q_{2}, q_{3}\right)$ & $\left(E C_{\infty}, q_{1}, q_{2}, q_{3}\right)$ \\
\hline 1 & $(1.041,0.6,1.0,1.0)$ & $(1.110,0.6,1.0,1.0)$ \\
2 & $(0.841,0.6,1.3,1.0)$ & $(0.958,0.6,1.3,1.0)$ \\
3 & $(0.844,0.6,1.3,1.0)$ & $(0.973,0.6,1.3,1.0)$ \\
4 & $(0.854,0.8,1.2,1.0)$ & $(0.988,0.8,1.2,1.0)$ \\
5 & $(0.862,0.8,1.2,1.0)$ & $(0.996,0.6,1.3,1.0)$ \\
10 & $(0.867,0.8,1.2,1.0)$ & $(0.999,0.8,1.2,1.0)$ \\
\hline
\end{tabular}

- $\delta$ and $E C_{\infty}$ : When $\delta$ changes from 1 to 2, its effect on $E C_{\infty}$ is obvious and the saved cost is up to $20 \%$. As $\delta$ increases, the unit cost for acquiring the signals is expensive and compensates the cost saving by the prediction signal. When $\delta$ is large enough, $E C_{\infty}$ is stable.

- $C_{l}$ and $q$. When unit production loss of soft failure $C_{l}$ is expensive, the preventive age threshold $q_{3}$ for soft components decreases and the soft components have more chances to be replaced at routine maintenance. As the set up cost $C_{s t}$ for reactive maintenance and down time cost $C_{h u}$ for maintenance action are expensive, such as $C_{s t}=C_{h u}=50$, the threshold for opportunistic at reactive maintenance $q_{1}$ is quite small.

Notice that each time when a prediction signal is received, the expected cost $E C_{i m}^{i}$ and $E C_{w d}^{i}$ have to be calculated and compared. Thus the calculation time of the programme is greater than the tolerance criteria, especially when the number of components is great.

\subsection{Comparison of the two criteria for opportunistic maintenance: tolerance criteria and expected cost criteria}

The parameter $\delta$ affects the approximation of the signal to the actual failure time. It is interesting to analyze the impact of $\delta$ when we compare the performance of the tolerance criteria and expected cost criteria policies. As $\delta$ decreases, the deviation between the prediction signal and the actual failure increase. Hence the expected cost criteria policy which considers both the signal and the age of the component performs better than the policy with design parameter $\varepsilon$ as shown by Figure 6 . As $\delta$ increases, the performance of the expected cost criteria becomes stable; the tolerance criteria policy performs better due to the timely prediction of potential hard failure by the prediction signal.

\section{Conclusion}

In this study, we considered a multi-component system with two failure modes in the framework of the system of system. Therefore the maintenance strategy of the individual system should be planned to accord with the overall planning of the 


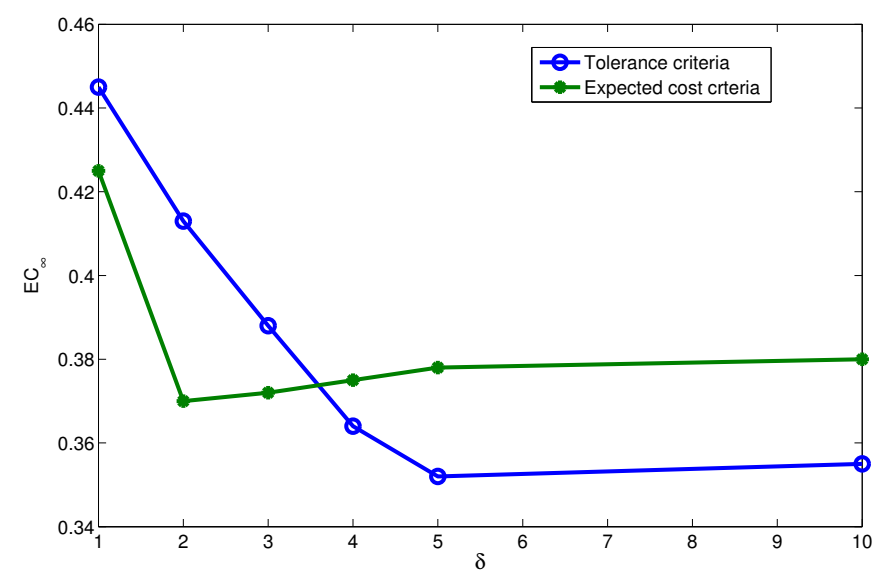

Figure 6: Comparison of tolerance criteria and expected cost criteria with the change of $\delta$ with $\eta=0.2$; for 3 hard components, $C$.f $=100$, $C_{\cdot p}=60 ;$ for one soft component $C_{\cdot f}=100, C_{\cdot p}=80 ; C_{u s}=10, C_{u h}=50, C_{s t}=50, C_{t}=5, C_{l}=10$

system of system. Besides, maintenance decision making with uncertainties is studied and the benefits of the monitoring information with different level of uncertainties are revealed. Based on the complex failure model, the uncertainties are represented by the random distribution of prediction signals of the hard component's failure. Then we proposed an integrated maintenance policy with three levels: routine, opportunistic and reactive maintenances corresponding to the system of systems level, the system level and the component level. For the reactive maintenance, two distinct criteria are considered: the designed tolerance and the expected cost criteria for the hard component. The mathematical expression of the cost is derived and the simulation procedure is presented. Then the impacts of uncertainties in decision making using the two criterions are studied and compared by numerical simulations.

In the future, in the framework of the integrated maintenance policy, more different life distributions could be considered. The interaction between hard failure and soft failure could be extended from independent to dependent in different ways. The maintenance action could be extended from the replacement to imperfect repair characterized by the change of the failure rate and the reduction of the age. Hence the life characters of the system will be more extensive to be suitable for more industrial and engineering practice.

[1] Dekker, R., Wildeman, R., van der Duyn Schouten, F.. A review of multi-component maintenance models with economic dependence. Mathematical Methods of Operations Research 1997;97(3):411-435.

[2] Nicolai, R., Dekker, R.. Optimal maintenance of multi-component systems: a review. London: Springer; 2008, p. $263-286$.

[3] Nowakowski, T., Werbińka, S.. On problems of multicomponent system maintenance modelling. International Journal of Automation and Computing 2008;6(4):364-378.

[4] Tsai, Y.T., Wang, K.S., Tsai, L.C.. A study of availability-centered preventive maintenance for multi-component systems. Reliability Engineering \& System Safety 2004;84(3):261-270.

[5] Gustavsson, E., Patriksson, M., Strömberg, A.B., Wojciechowski, A., Önnheim, M.. Preventive maintenance scheduling of multicomponent systems with interval costs. Computers \& Industrial Engineering 2014;76:390-400.

[6] Castanier, B., Grall, A., Bérenguer, C.. A condition-based maintenance policy with non-periodic inspections for a two-unit series system. Reliability Engineering \& System Safety 2005;87(1):109-120.

[7] Laggoune, R., Chateauneuf, A., Aissani, D.. Opportunistic policy for optimal preventive maintenance of a multi-component system in continuous operating units. Computers \& Chemical Engineering 2009;33(9):1499-1510.

[8] Jia, Q.. A structural property of optimal policies for multi-component maintenance problems. Automation Science and Engineering, IEEE Transactions on 2010;7(3):671-680.

[9] Moghaddam, K.S., Usher, J.S.. A new multi-objective optimization model for preventive maintenance and replacement scheduling of multi-component systems. Engineering Optimization 2011;43(7):701-719.

[10] Zhang, Z., Wu, S., Li, B., Lee, S.. (n,N) type maintenance policy for multi-component systems with failure interactions. International 
Journal of Systems Science 2015;46(6):1051-1064.

[11] Zhou, X., Huang, K., Xi, L., Lee, J.. Preventive maintenance modeling for multi-component systems with considering stochastic failures and disassembly sequence. Reliability Engineering \& System Safety 2015;142:231-237.

[12] Vu, H.C., Do, P., Barros, A., Bérenguer, C.. Maintenance grouping strategy for multi-component systems with dynamic contexts. Reliability Engineering \& System Safety 2014;132:233-249

[13] Zhang, N., Yang, Q.. Optimal maintenance planning for repairable multi-component systems subject to dependent competing risks. IIE Transactions 2015;47(5):521-532.

[14] Zhu, Q., Peng, H., van Houtum, G.J.. A condition-based maintenance policy for multi-component systems with a high maintenance setup cost. OR Spectrum 2015;37(4):1007-1035.

[15] Lin, D., Zuo, M., Yam, R.. Sequential imperfect preventive maintenance models with two categories of failure modes. Naval Research Logistics 2001;48(2):172-183.

[16] Zequeira, R., Bérenguer, C.. Periodic imperfect preventive maintenance with two categories of competing failure modes. Reliability Engineering \& System Safety 2006;91(4):460-468.

[17] Zhang, Y., Wang, G.. An extended replacement policy for a deteriorating system with multi-failure modes. Applied Mathematics and Computation 2011;218(5):1820-1830.

[18] Castro, I.. A model of imperfect preventive maintenance with dependent failure modes. European Journal of Operational Research $2009 ; 196(1): 217-224$.

[19] Wang, W., Banjevic, D., Pecht, M.. A multi-component and multi-failure mode inspection model based on the delay time concept. European Journal of Operational Research 2010;95(8):912-920.

[20] Ding, F., Tian, Z.. Opportunistic maintenance for wind farms considering multi-level imperfect maintenance thresholds. Renewable Energy 2012;45:175-182.

[21] Hong, H., Zhou, W., Zhang, S., Ye, W.. Optimal condition-based maintenance decisions for systems with dependent stochastic degradation of components. Reliability Engineering \& System Safety 2014;121:276-288.

[22] Jardine, A., Lin, D., Banjevic, D.. A review on machinery diagnostics and prognostics implementing condition-based maintenance. Mechanical Systems and Signal Processing 2006;20(7):1483-1510.

[23] Sikorska, J., Hodkiewicz, M., Ma, L.. Prognostic modelling options for remaining useful life estimation by industry. Mechanical Systems and Signal Processing 2011;25(5):1803-1836.

[24] Saxena, A., Celaya, J., Saha, B., Saha, S., Goebel, K.. Metrics for offline evaluation of prognostic performance. International journal of prognostics and health management 2010;1(1):1-20.

[25] Yan, J., Koç, M., Lee, J.. A prognostic algorithm for machine performance assessment and its application. Production Planning \& Control 2004;15(8):796-801.

[26] Yu, G., Qiu, H., Djurdjanovic, D., Lee, J.. Feature signature prediction of a boring process using neural network modeling with confidence bounds. International Journal of Advanced Manufacturing Technology 2006;30(7-8):614-621.

[27] Lorton, A., Fouladirad, M., Grall, A.. A methodology for probabilistic model-based prognosis. International journal of prognostics and health management 2013;225(3):443-454.

[28] McKone, K., Weiss, E.. Guidelines for implementing predictive maintenance. Production and Operations Management 2002;11(2):109124.

[29] Zequeira, R., Bérenguer, C.. Optimal inspection policies with predictive and preventive maintenance. Engineering Optimization 2005;37(5):541-550.

[30] Bedford, T., Dewan, I., Meilijson, I., Zitrou, A.. The signal model: A model for competing risks of opportunistic maintenance. European Journal of Operational Research 2011;214(3):665-673.

[31] Van Horenbeek, A., Pintelon, L.. A dynamic predictive maintenance policy for complex multi-component systems. Reliability Engineering \& System Safety 2013;120:39-50.

[32] Taghipour, S., Banjevic, D.. Optimal inspection interval for a system under periodic and opportunistic inspections. IIE Transactions 2012a;44(11):932-948.

[33] Taghipour, S., Banjevic, D.. Optimal inspection of a complex system subject to periodic and opportunistic inspections and preventive replacements. European Journal of Operational Research 2012b;220:649-660.

[34] Tavner, P., Xiang, J., Spinato, F.. Reliability analysis for wind turbines. Wind Energy 2007;10(1):1-18.

[35] Johan, R., Bertling, L.. Survey of failures in wind power systems with focus on swedish wind power plants during 1997-2005. Energy Conversion, IEEE Transactions on 2007;22:167-173.

[36] Spinato, F., Tavner, P., Van Bussel, G., Koutoulakos, E.. Reliability of wind turbine subassemblies. Renewable Power Generation, IET 2009;3(4):387-401. 


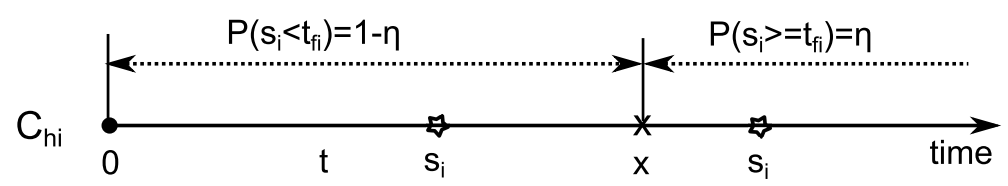

Figure A.7: The possible locations of the prediction signal of $C_{h i}$ given the failure occurs at x

\section{Appendix A. Cost caused by the hard components}

In this section we begin with the reactive maintenance policy without delay, i.e, once a prediction signal is received, the reactive maintenance should be executed immediately. The tolerance criteria and the excepted cost criteria become invalid.

Let $D_{l i}$ be the design life of $C_{h i}$. Denote $l_{i}^{j}=q_{j} D_{l i}$ the preventive replacement age threshold for $C_{h i}$ at routine maintenance, $j \in\{1,2,3\}$. Let $Y_{k}$ be the $k$ th replacement time interval for the hard components. $Y_{1}$ is competed by the life time $t_{f i}$, the corresponding prediction signal $s_{i}$ and the age threshold $l_{i}^{2}, i=1, \ldots, m_{1}$ at routine maintenance. Let $J_{i}(t)=\left\lceil\frac{t}{\tau}\right\rceil$ be the number of routine periods that hard component $C_{h i}$ gets through without prediction signal and failure during time interval $t$.

\section{Appendix A.1. Probability associated to the different maintenance actions}

The cost caused by the maintenance of the hard components during the first cycle $Y_{1}$ consists of three parts:

1. the cost caused by preventive maintenance (PM) which means that a prediction signal occurs before the actual hard failure,

2. the cost caused by corrective maintenance $(\mathrm{CM})$ which means that a hard failure occurs without a prediction to warn it,

3. and the cost caused by opportunistic maintenance $(\mathrm{OM})$ which means that the age threshold which decides the OM is reached.

For component $C_{h i}$, its first maintenance may be caused by a PM, a CM or an $\mathrm{OM}$ and the corresponding replacement cycles are denoted by $A_{i, 1}^{P}, A_{i, 1}^{C}$ and $A_{i, 1}^{O}$ respectively. Let $A_{i, j}$ be the $j$ th replacement cycle of component $C_{h i}$. Hence we have

$$
\begin{gathered}
\mathbb{P}\left(A_{i, 1}^{P}<t\right)=\mathbb{P}\left(s_{i} \leq t, t_{f i}>t\right) \\
\mathbb{P}\left(A_{i, 1}^{C}<t\right)=\mathbb{P}\left(s_{i}>t, t_{f i}<t\right) \\
\mathbb{P}\left(A_{i, 1}^{O}<t\right)=\mathbb{P}\left(s_{i}>t, t_{f i}>t, l_{i}^{1}<\xi_{i}(t), l_{i}^{2}<\xi_{i}\left(J_{i}(t) \tau\right)\right)
\end{gathered}
$$

and by definition

$$
\mathbb{P}\left(A_{i, 1}<t\right)=\mathbb{P}\left(A_{i, 1}^{P}<t\right)+\mathbb{P}\left(A_{i, 1}^{C}<t\right)+\mathbb{P}\left(A_{i, 1}^{O}<t\right)
$$

Recall that $C_{h p}(i), C_{h f}(i)$ and $C_{h u}$ are respectively the preventive replacement, corrective replacement and downtime cost of the hard component $C_{h i}$. The cost $C_{t}$ is incurred by a routine maintenance. Denote $C_{i m}^{h}(t)$ the total cost caused by hard components during time interval $t$. The random variables $A_{i, j}, j=1,2, \cdots$, are independent and identically distributed, thus we have

$$
C_{i m}^{h}(t)=\sum_{i=1}^{m_{1}}\left(N^{p, i}(t)+N^{o, i}(t)\right) C_{h p}(i)+N^{c, i}(t)\left(C_{h f}(i)+C_{s t}\right)+C_{t}+C_{h u}
$$


and

$$
N^{p, i}(t)=\sum_{j=1}^{\infty} \mathbb{1}_{\left\{A_{i, j}^{P}<t\right\}}, \quad N^{o, i}(t)=\sum_{j=1}^{\infty} \mathbb{1}_{\left\{A_{i, j}^{o}<t\right\}}, \quad N^{c, i}(t)=\sum_{j=1}^{\infty} \mathbb{1}_{\left\{A_{i, j}^{c}<t\right\}}
$$

The downtime $\operatorname{cost} C_{h u}$ is counted once, because when the reactive maintenance is triggered, the whole system should be shutdown and the downtime cost $C_{h u}$ is incurred by the component whose prediction signal occurs first or which fails first. Hence for the other components which take the opportunity to be maintained, $C_{h u}$ is actually saved and that's why we consider opportunistic maintenance actions. The average total cost caused by hard component after $n$ maintenances at $T_{n}$ is expressed as follows:

$$
\mathbb{E}\left(C_{i m}^{h}\left(T_{n}\right)\right)=\sum_{k=1}^{n} \mathbb{E}\left(C_{i m}^{h}\left(Y_{k}\right)\right)=\sum_{k=1}^{n} \int_{0}^{\infty} \mathbb{E}\left(C_{i m}^{h}(t)\right) d \mathbb{P}\left(Y_{k}<t\right)
$$

In the remainder of this section one derives the following quantities: $\mathbb{P}\left(s_{i}>t, t_{f i}<t\right), \mathbb{P}\left(s_{i}<t, t_{f i}>t\right), \mathbb{P}\left(Y_{k}<t\right)$, $\mathbb{P}\left(\xi_{i}(\cdot)<t\right)$.

\section{Appendix A.2. The probability associated to the prediction signal}

According to the definition of the conditional signal and Figure A.7 which explains the possible locations of the prediction signal, there are only two possibilities for the position of the prediction signal. The first possibility is that the prediction signal occurs before the actual hard failure, and we know that the prediction accuracy of the prediction signal is $\eta$, i.e., $\mathbb{P}\left(s_{i}<t_{f i}\right)=1-\eta$. The second possibility is that the prediction signal does not occur before the hard failure with the probability $\mathbb{P}\left(s_{i} \geq t_{f i}\right)=\eta$. Therefore, for a given failure time the probability that the signal occurs in a given interval before the failure is as follows:

$$
\begin{aligned}
\mathbb{P}\left(t<s_{i}<x \mid t_{f i}=x\right) & =\mathbb{P}\left(0<s_{i}<x \mid t_{f i}=x\right)-\mathbb{P}\left(0<s_{i}<t \mid t_{f i}=x\right) \\
& =1-F_{S_{i}}(t \mid x), \quad 0 \leq t \leq x
\end{aligned}
$$

Therefore,

$$
\begin{aligned}
\mathbb{P}\left(s_{i}>t \mid t_{f i}>t\right)= & \int_{t}^{\infty} \mathbb{P}\left(s_{i}>t \mid t_{f i}=x\right) f_{i}(x) d x \\
= & \int_{t}^{\infty}\left[\mathbb{P}\left(s_{i}>t \mid t_{f i}=x, s_{i} \geq t_{f i}\right) \mathbb{P}\left(s_{i} \geq t_{f i}\right)\right. \\
& \left.+\mathbb{P}\left(t<s_{i}<x \mid t_{f i}=x, s_{i}<t_{f i}\right) \mathbb{P}\left(s_{i}<t_{f i}\right)\right] f_{i}(x) d x \\
= & \int_{t}^{\infty}\left[\eta+(1-\eta)\left(1-F_{S_{i}}(t \mid x)\right)\right] f_{i}(x) d x
\end{aligned}
$$

Appendix A.3. The probability associated to the maintenance cycles of the system

Let us define the following random variables:

- $T_{k}=\sum_{j=1}^{k} Y_{k}$ : the time of the $k$ th maintenance of the system. $k$ is the number of maintenance of the system (caused by hard components, but we don't distinguish which component causes the maintenance by the definition of $Y_{k}$ ).

- $A_{i, j}$ : the $j$ th replacement cycle of component $C_{h i}$.

- $a_{i, n_{i}}=\sum_{j=1}^{n_{i}} A_{i, j}$ : the time of the $n_{i}$ th replacement of component $C_{h i}$.

- $N_{i}(t)=\sum_{j=1}^{\infty} \mathbb{1}_{\left\{a_{i, j} \leq t\right\}}$ 


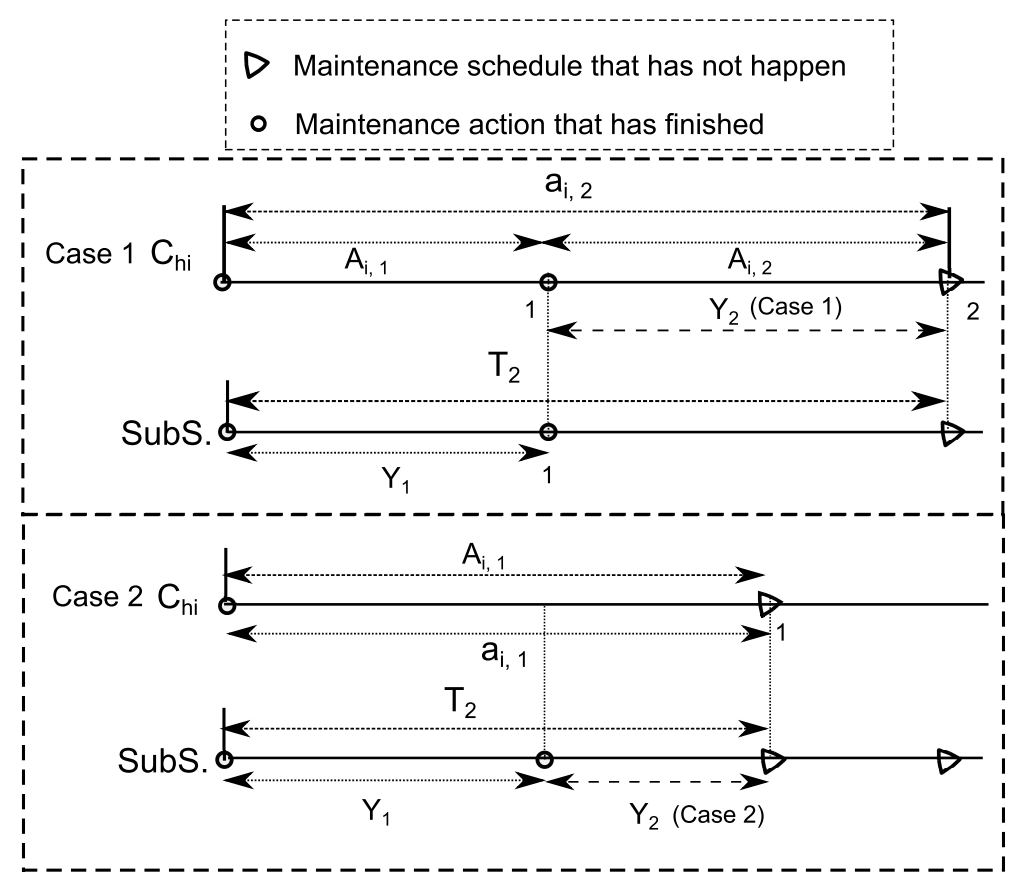

Figure A.8: Two possible renewal process of component $C_{h i}$ after first reactive maintenance of the system. For Case 1, component $C_{h i}$ is renewed at $Y_{1}$ and then it is renewed again after a duration of $A_{i, 2}$. Thus $Y_{2}=a_{i, 2}-Y_{1}$ for the whole system. For Case 2, component $C_{h i}$ survives at $Y_{1}$. It may be renewed after a period $A_{i, 1}$ and thus $Y_{2}=a_{i, 1}-Y_{1}$.

By using Eq. (A.8), the probability distribution function associated to the first replacement time interval $Y_{1}$ can be calculated as follows,

$$
\begin{aligned}
\mathbb{P}\left(Y_{1}>t\right) & =\mathbb{P}\left(\min \left(t_{f 1}, s_{1}, l_{1}^{2}, \ldots, t_{f m_{1}}, s_{m_{1}}, l_{m_{1}}^{2}\right)>t\right) \\
& =\prod_{i=1}^{m_{1}} \mathbb{P}\left(t_{f i}>t, s_{i}>t\right) \mathbb{P}\left(l_{i}^{2}>\xi_{i}\left(J_{i}(t) \tau\right)\right) \\
& =\prod_{i=1}^{m_{1}} \mathbb{P}\left(s_{i}>t \mid t_{f i}>t\right) \mathbb{P}\left(t_{f i}>t\right) \mathbb{P}\left(l_{i}^{2}>\xi_{i}\left(J_{i}(t) \tau\right)\right)
\end{aligned}
$$

Figure A.8 shows a schema of the possible realizations of $Y_{2}$ (the second maintenance time interval of the system). SubS denotes the subsystem consisting of the hard components exclusive of $C_{h i}$. For component $C_{h i}$ there are two possibilities after $Y_{1}$ :

- Case 1: The component $C_{h i}$ has been replaced at $Y_{1}$. The maintenance cycle $Y_{2}$ is equal to $a_{i, 2}-T_{1}$.

- Case 2: The component $C_{h i}$ has survived at $Y_{1}$. The maintenance cycle $Y_{2}$ is equal to $a_{i, 1}-T_{1}$.

Considering all the $m_{1}-1$ hard components, the following probability distribution can be deduced:

$$
\begin{aligned}
& \mathbb{P}\left(Y_{2}>t\right)=\mathbb{P}\left(\min \left(a_{1, N_{1}\left(T_{1}\right)+1}-T_{1}, \ldots, a_{m_{1}, N_{m_{1}}\left(T_{1}\right)+1}-T_{1}\right)>t\right) \\
&= \prod_{i=1}^{m_{1}} \int_{0}^{\infty} \sum_{j=0}^{1} \mathbb{P}\left(a_{i, j+1}-T_{1}>t \mid T_{1}, N_{i}\left(T_{1}\right)=j\right) \mathbb{P}\left(N_{i}\left(T_{1}\right)=j\right) d\left(\mathbb{P}\left(T_{1} \leq x\right)\right) \\
&= \prod_{i=1}^{m_{1}} \int_{0}^{\infty}\left[\mathbb{P}\left(a_{i, 1}-T_{1}>t \mid T_{1} \leq x\right) \mathbb{P}\left(N_{i}\left(T_{1}\right)=0\right)+\right. \\
&\left.\mathbb{P}\left(a_{i, 2}-T_{1}>t \mid T_{1} \leq x\right) \mathbb{P}\left(N_{i}\left(T_{1}\right)=1\right)\right] d\left(\mathbb{P}\left(T_{1} \leq x\right)\right)
\end{aligned}
$$

where $\mathbb{P}\left(T_{1} \leq x\right)=\mathbb{P}\left(Y_{1} \leq x\right)$ is given by equation (A.9). 


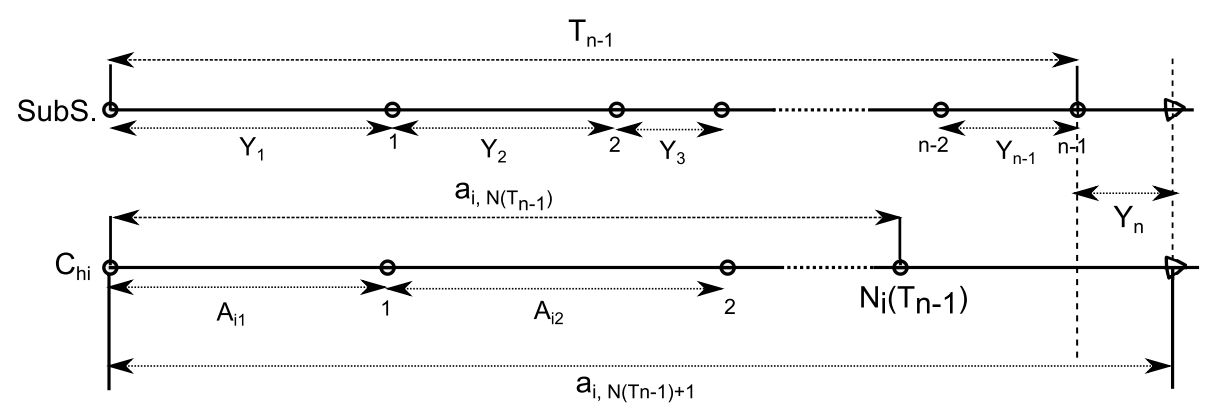

Figure A.9: Replacement process of component $C_{h i}$ after $(n-1)$ th reactive maintenance of the system. For component $C_{h i}$, its replacement time may contribute to the replacement process of the whole system, but it is different from the latter.

In the same way we can calculate the distribution of $Y_{n}$. See Figure A.9 for an example of $Y_{n}$, the survival function of $Y_{n}$ can be calculated recursively as follows:

$$
\begin{aligned}
& \mathbb{P}\left(Y_{n}>t\right)=\mathbb{P}\left(\min \left(a_{1, N_{1}\left(T_{n-1}\right)+1}-T_{n-1}, \ldots, a_{m_{1}, N_{m_{1}}\left(T_{n-1}\right)+1}-T_{n-1}\right)>t\right) \\
& =\prod_{i=1}^{m_{1}} \int_{0}^{\infty} \sum_{j=0}^{n-1} \mathbb{P}\left(a_{i, N_{i}\left(T_{n-1}\right)+1}-T_{n-1}>t \mid T_{n-1}, N_{i}\left(T_{n-1}\right)=j\right) \mathbb{P}\left(N_{i}\left(T_{n-1}\right)=j\right) \\
& d\left(\mathbb{P}\left(T_{n-1} \leq x\right)\right)
\end{aligned}
$$

where

$$
\begin{gathered}
\mathbb{P}\left(T_{n-1} \leq x\right)=\mathbb{P}\left(\sum_{j=1}^{n-1} Y_{j} \leq x\right) \\
\mathbb{E}\left(Y_{n}\right)=\int_{0}^{\infty} \mathbb{P}\left(Y_{n}>y\right) d y
\end{gathered}
$$

To calculate Eq. (A.10) and Eq. (A.11), the probabilities related to $a_{i, j}$ and consequently $A_{i, j}$ should be evaluated. As the failures (respectively signals) of different components are independent of each other and after replacement the component $C_{h i}$ starts as new, then $a_{i, h_{i}}$ is a renewal process and $N_{i}(t)$ is the renewal function.

For $C_{h i}$, the design life $D_{l i}$ is constant and $l_{i}^{1}=q_{1} D_{l i}, q_{1}>0$ is designed to decide whether or not the component $C_{h i}$ is opportunistically maintained while it is functioning during a system reactive maintenance. Recall that for a fixed $q_{1}$, the parameter $l_{i}^{1}$ is constant.

The inequality $A_{i, j}>t$ implies that the following four events are occurred: after being functional during $t$ since the last maintenance (the $(j-1)$ th replacement for $C_{h i}$ ),

- Event 1: $C_{h i}$ is not failed;

- Event 2: $C_{h i}$ does not show a prediction signal;

- Event 3: its age does not reach the threshold $l_{i}^{1}$ at the reactive maintenance triggered by the other hard components;

- Event 4: its age does not reach the threshold $l_{i}^{2}$ at the $J_{i}\left(a_{i, j-1}+t\right)$ th routine maintenance of the system.

The $A_{i, j}$ are identically distributed and according to the four events, we have

$$
\begin{aligned}
\mathbb{P}\left(A_{i, j}>t\right) & =\mathbb{P}\left(A_{i, 1}>t\right) \\
& =\mathbb{P}\left(t_{f 1}>t, s_{1}>t, l_{i}^{1}>\xi_{i}(t), l_{i}^{2}>\xi_{i}\left(J_{i}\left(a_{i, j-1}+t\right) \tau\right)\right) \\
& =\mathbb{P}\left(s_{1}>t \mid t_{f 1}>t\right) \mathbb{P}\left(t_{f 1}>t\right) \mathbb{P}\left(l_{i}^{1}>\xi_{i}(t)\right) \mathbb{P}\left(l_{i}^{2}>\xi_{i}\left(J_{i}\left(a_{i, j-1}+t\right) \tau\right)\right)
\end{aligned}
$$


The renewal function has the following probability distribution:

$$
\mathbb{P}\left(N_{i}(t) \geq n\right)=\mathbb{P}\left(a_{i, n} \leq t\right)=F_{A_{i, 1}}^{(n)}(t)
$$

where $F_{A_{i, 1}}(t)$ is the cumulative distribution function (cdf) of $A_{i, 1}$ and $F_{A_{i, 1}}^{(n)}(\cdot)$ denotes the $n$-fold convolution of $F_{A_{i, 1}}(\cdot)$. Since

$$
\mathbb{P}\left(N_{i}(t)=n\right)=\mathbb{P}\left(a_{i, n+1}>t\right)-\mathbb{P}\left(a_{i, n}>t\right),
$$

therefore,

$$
\mathbb{P}\left(a_{i, N_{i}(t)}>h\right)=\sum_{n=0}^{\infty} \mathbb{P}\left(\sum_{j=1}^{n} A_{i, j}>h \mid N_{i}(t)=n\right) \mathbb{P}\left(N_{i}(t)=n\right) .
$$

\section{Appendix B. Cost caused by the soft components}

The soft components are inspected both at reactive maintenance and at the routine maintenance times with time interval $\tau$. Hence the cycle of the maintenance of soft component composes of both $\left\{Y_{k}, k \geq 1\right\}$ and $\{n \tau, n \geq 1\}$ based on its age threshold and life.

Let us define the following random variables:

- $B_{i, j}$ : the $j$ th replacement cycle of soft component $C_{s i}$.

- $b_{i, k}=\sum_{j=1}^{k} B_{i, j}$.

- $N^{S}(t)=\sum_{j=0}^{\infty} \mathbb{1}_{\left\{T_{j}<t\right\}}$ the counting process of the reactive maintenance actions of the system, (number of reactive maintenances before $t$ ).

- $T_{N^{S}(t)}$ : the time of the $N^{S}(t)$-th reactive maintenance actions of the system

- $N^{\tau}(t)=\sum_{j=0}^{\infty} \mathbb{1}_{\{j \tau<t\}}$ the counting process of the routine maintenance actions of the system.

The soft component is maintained if one of the following events occurs:

- The component is failed before a reactive maintenance.

- The component is failed before a routine maintenance.

- The next reactive maintenance after the last maintenance is planned is longer than $l_{i}$.

- The next routine maintenance time is planned after $l_{i}$.

In figures B.10, B.11, B.12, B.13, examples of the replacement cycle of soft component $C_{s i}$ are given. $H_{S u b s .}$ denotes the subsystem of hard components.

Appendix B.1. Probabilities costs associated to maintenance cycles

For the first cycle $B_{i, 1}$ of the soft component $C_{s i}$, for simplification of notation and derivation, let $l_{i}^{1}=l_{i}^{2}=l_{i}$, we have

$$
\begin{aligned}
\mathbb{P}\left(B_{i, 1}>t\right) & =\mathbb{P}\left(t_{f i}>T_{N^{S}(t)}, t_{f i}>N^{\tau}(t) \tau, l_{i}>T_{N^{S}(t)}, l_{i}>N^{\tau}(t) \tau\right) \\
& =\mathbb{P}\left(\min \left(t_{f i}, l_{i}\right)>\max \left(T_{N^{S}(t)}, N^{\tau}(t) \tau\right)\right)
\end{aligned}
$$

Similarly,

$$
\mathbb{P}\left(B_{i, 2}>t\right)=\mathbb{P}\left(\min \left(t_{f i}, l_{i}\right)>\max \left(T_{N^{S}\left(t+B_{i, 1}\right)}-B_{i, 1}, N^{\tau}(t) \tau\right)\right)
$$




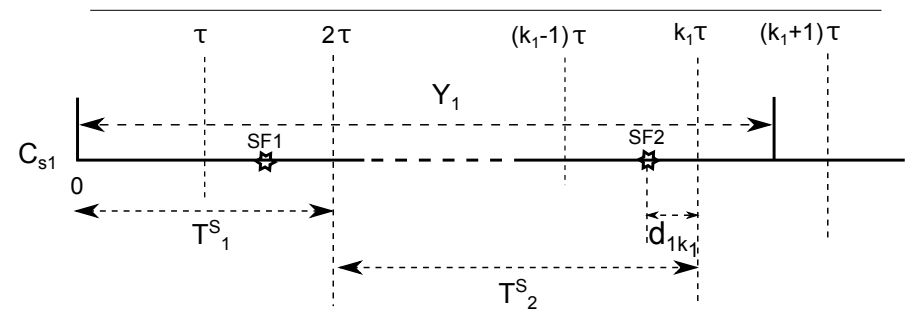

Figure B.10: The soft failure duration during $Y_{1} . S F 1$ and $S F 2$ denote the times when the first and second soft failures occur. $T_{i}^{s}, i=1,2$ denote the replacement cycle of soft component. $d_{1 K_{1}}$ denotes the downtime during $K_{1}$ th routine maintenance period in $Y_{1}$.

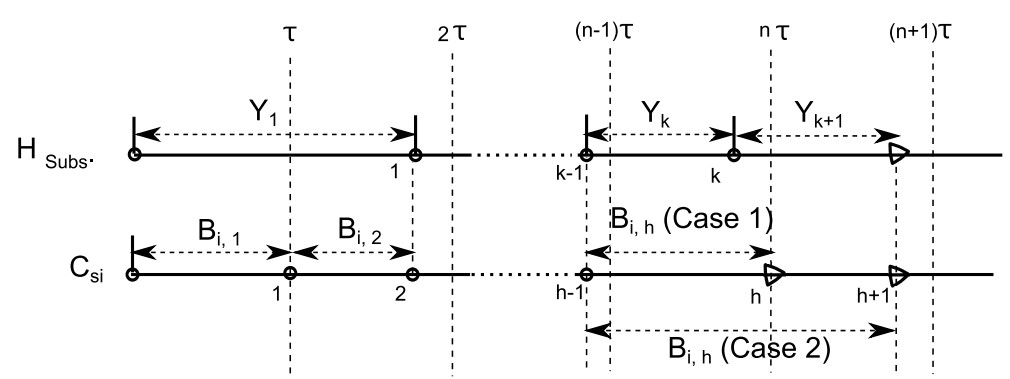

Figure B.11: Replacement process of component $C_{s i}$, where $B_{i, h}$ denotes the $h$ th maintenance of soft component $C_{s i}$ and $Y_{k}$ denotes the $k$ th maintenance of the system. $B_{i, h}$ is decided by either $n \tau$ ( $n$th routine maintenance) or $Y_{k+1}$ (reactive maintenance caused by hard components)

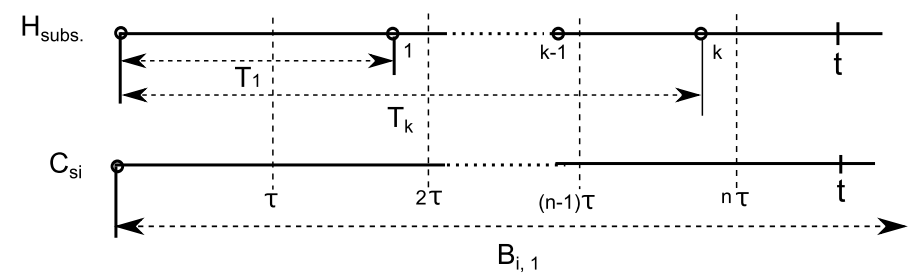

Figure B.12: The first replacement cycle of component $C_{s i}$. The component $C_{s i}$ could be replaced either by routine maintenance or opportunistic maintenance triggered by reactive maintenance of hard components.

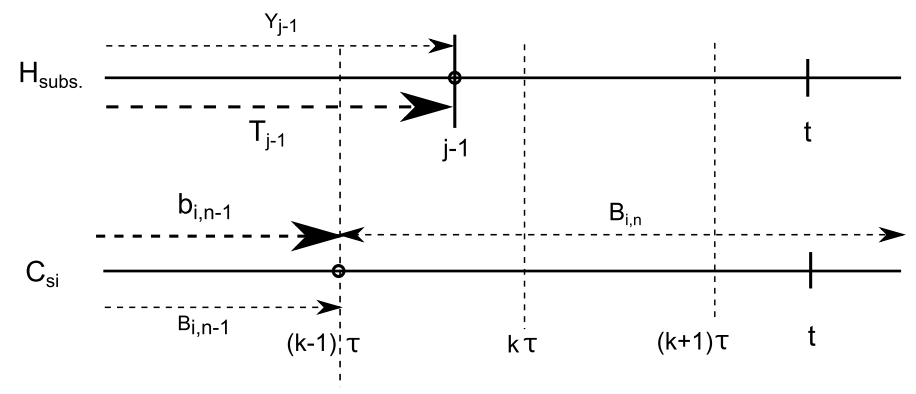

Figure B.13: The possible $n$th renewal cycle of component $C_{s i}$. For $H_{\text {subs. }}$. it may experience $j-1$ th maintenance, while for soft component $C_{s i}$, it has been renewed $n-1$ times. 
For the $n$th replacement, it depends on $b_{i, n-1}$ and we have

$$
\mathbb{P}\left(B_{i, n}>t\right)=\mathbb{P}\left(\min \left(t_{f i}, l_{i}\right)>\max \left(T_{N^{S}\left(t+b_{i, n-1}\right)}-b_{i, n-1}, N^{\tau}(t) \tau\right)\right)
$$

The challenge is that even we calculate the probability distribution function of $\left\{B_{i, n}, n \in R^{+}\right\}$recursively, it is still hard to distinguish whether the maintenance of the soft component occurs at a reactive maintenance time or at a routine maintenance time. If it occurs at the reactive maintenance, the down time cost will be saved by the maintenance of hard components. In order to simplify the analysis, assume that the time duration of the replacement of the soft component is negligible, i.e, $C_{s u}=0$.

\section{Appendix B.2. Probabilities associated to each maintenance operation}

Now we focus on the production loss during the soft failure. As the soft failure is hidden and the maintenance can only happen at the reactive and routine maintenance, the soft failure can only be fixed at the next reactive maintenance or routine maintenance, whichever occurs first. Recall that $Y_{n}$ denotes the time interval of $n$th maintenance of the system caused by hard components. The following notations are considered.

- $K_{n}=\left\lceil\frac{Y_{n}}{\tau}\right\rceil$ : the maximum number of routine maintenances during $Y_{n}$, where $\lceil x\rceil=\max \{n \in \mathbb{N} \mid n \leq x\}$.

- $k_{n}=\sum_{i=1}^{n} K_{i}$ : the number of routine maintenance during $T_{n}$.

- $N^{f}(t)$ : the number of soft failures during the period $t$

- $\xi_{i}(t)$ : the age of the soft component $C_{s i}$ at time $t . \xi_{i}(0)=0$ and after replacement at $t, \xi_{i}\left(t^{+}\right)=0$.

Figure B.10 gives an example of the soft failure during $Y_{1}$. The first soft failure occurs between $\tau$ and $2 \tau$ and is fixed at $2 \tau$. For further simplifications, we consider a constant production loss $C_{l}$. If the production loss is a constant for each soft failure, we only need to know the times of the soft failures.

We start with $m_{2}=1$, i.e, one soft component. The number of soft failures $N^{f}\left(Y_{1}\right)$ during $Y_{1}=T_{1}$ is defined as follows

$$
N^{f}\left(Y_{1}\right)=\sum_{j=1}^{K_{1}} \mathbb{1}_{\left\{\xi_{i}(j \tau)=0\right\}}+\mathbb{1}_{\left\{t_{s f}<Y_{1}-K_{1} \tau+\xi_{i}\left(K_{1} \tau\right)\right\}}
$$

where $\xi_{i}(j \tau)=0$ implies a replacement at $j \tau$ and $t_{s f}<Y_{1}-K_{1} \tau+\xi_{i}\left(K_{1} \tau\right)$ implies the component does not survive at the last incomplete routine period included in $Y_{1}$. Since

$$
\begin{aligned}
\mathbb{P}\left(K_{1}=k\right) & =\mathbb{P}\left(\left\lceil\frac{Y_{1}}{\tau}\right\rceil=k\right)=\mathbb{P}\left(k \tau<Y_{1}<(k+1) \tau\right) \\
& =\mathbb{P}\left(Y_{1}<(k+1) \tau\right)-\mathbb{P}\left(Y_{1}<k \tau\right)
\end{aligned}
$$

Therefore,

$$
\mathbb{E}\left(N^{f}\left(Y_{1}\right)\right)=\sum_{k=1}^{\infty}\left(\sum_{j=1}^{k} \mathbb{P}\left(\xi_{i}(j \tau)=0\right)+\mathbb{P}\left(t_{s f}<Y_{1}-k \tau+\xi_{i}(k \tau)\right)\right) \mathbb{P}\left(K_{1}=k\right)
$$

Since the soft component is inspected at each routine maintenance, the soft failure must be revealed in time interval $\tau$. Thus the first part of Eq. (B.6) can be calculated as follows,

$$
\mathbb{P}\left(\xi_{i}(j \tau)=0\right)=\mathbb{P}\left((j-1) \tau<t_{s f}<j \tau\right)
$$




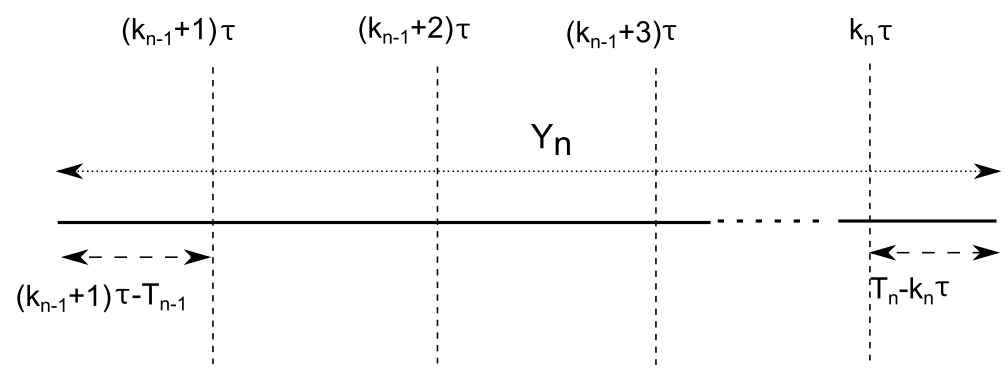

Figure B.14: The possible soft failure duration during $Y_{n}$. It consists of three parts in $Y_{n}$ : the period that is partly contained in $Y_{n-1}$ and $Y_{n}:\left(K_{n-1}+1\right) \tau-T_{n-1}$; the periods contained entirely in $Y_{n}$; the period that is partly contained in $Y_{n}$ and $Y_{n+1}: T_{n}-K_{n} \tau$.

The second part of Eq. (B.6) is calculated as follows. Since before $Y_{1}$ the only replacement can occur at routine maintenance times, therefore the soft component age $\xi_{i}$ at a routine maintenance time is a multiple of $\tau$. Consequently,

$$
\begin{aligned}
& \mathbb{P}\left(t_{s f}<Y_{1}-k \tau+\xi_{i}(k \tau)\right)=\sum_{l=0}^{k} \mathbb{P}\left(t_{s f}<Y_{1}-(k-l) \tau \mid \xi_{i}(k \tau)=l \tau\right) \mathbb{P}\left(\xi_{i}(k \tau)=l \tau\right) \\
& \quad=\int_{0}^{\infty} \sum_{l=0}^{k} \mathbb{P}\left(t_{s f}<Y_{1}-(k-l) \tau \mid \xi_{i}(k \tau)=l \tau, Y_{1}=y\right) \mathbb{P}\left(\xi_{i}(k \tau)=l \tau\right) f_{Y_{1}}(y) d y \\
& \quad=\int_{0}^{\infty} \sum_{l=0}^{k} \mathbb{P}\left(t_{s f}<Y_{1}-(k-l) \tau \mid \xi_{i}(k \tau)=l \tau, Y_{1}=y\right) \mathbb{P}\left(t_{s f}>l \tau\right) f_{Y_{1}}(y) d y
\end{aligned}
$$

where $Y_{1}$ is defined by Eq. (A.9).

Recall $K_{i}=\left\lceil\frac{Y_{i}}{\tau}\right\rceil$ be the maximum routine maintenance times during $Y_{i}$ and $k_{n}=\sum_{i=1}^{n} K_{i}$. Similarly as it is shown in Fig B.14, the number of soft failures $N^{f}\left(Y_{n}\right)$ during $Y_{n}$ is as follows:

$$
N^{f}\left(Y_{n}\right)=\underbrace{\sum_{j=k_{n-1}+1}^{k_{n}} \mathbb{1}_{\left\{\xi_{i}(j \tau)=0\right\}}}_{\text {Part } I}+\underbrace{\mathbb{1}_{\left\{t_{s f}<T_{n}-k_{n} \tau+\xi_{i}\left(k_{n} \tau\right)\right\}}}_{\text {Part II }}
$$

where

- Part $I$ corresponds to the soft failures detected and maintained at routine maintenance times.

- Part $I I$ the failure occurs after the last routine maintenance and before $T_{n}$.

The expected number of soft failure is calculated as follows:

$$
\mathbb{E}\left(N^{f}\left(Y_{n}\right)\right)=\sum_{k=1}^{\infty} \sum_{j=k_{n-1}+1}^{k_{n}} \mathbb{P}\left(\xi_{i}(j \tau)=0\right)+\mathbb{P}\left(t_{s f}<T_{n}-k \tau+\xi_{i}(k \tau)\right) \mathbb{P}\left(k_{n}=k\right)
$$

where $\mathbb{P}\left(\xi_{i}(j \tau)=0\right)$ is given by Eq.(B.7) and

$$
\mathbb{P}\left(k_{n}=k\right)=\mathbb{P}\left(\sum_{i=1}^{n} K_{i}=k\right)=\mathbb{P}^{(n)}\left(K_{i}=k\right),
$$

with $\mathbb{P}^{(n)}$ the $n$ convolution of the probability and similarly to Eq.(B.5)

$$
\mathbb{P}\left(K_{n}=k\right)=\mathbb{P}\left(Y_{y}<(k+1) \tau\right)-\mathbb{P}\left(Y_{y}<k \tau\right)
$$

Moreover,

$$
\begin{aligned}
\mathbb{P}\left(t_{s f}<T_{n}-k \tau\right. & \left.+\xi_{i}(k \tau)\right)=\int_{\mathbb{R}} \mathbb{P}\left(t_{s f}<t-k \tau+\xi_{i}(k \tau)\right) d \mathbb{P}\left(T_{n}<t\right) \\
& =\sum_{l=1}^{K_{n}} \int_{\mathbb{R}} \mathbb{P}\left(t_{s f}<t-k \tau+l \tau\right) d \mathbb{P}\left(T_{n}<t\right) \mathbb{P}\left(\xi_{i}(k \tau)=l \tau\right)
\end{aligned}
$$


where

$$
\mathbb{P}\left(\xi_{i}(k \tau)=l \tau\right)=\mathbb{P}\left((k-l-1) \tau<t_{s f}<(k-l) \tau\right)
$$

Besides the number of the soft failure $N_{n}^{f}$, the number of preventive maintenance $N_{n}^{p}$ during $Y_{n}$ should be calculated. Analogically as the $N^{f}$, we have

$$
\begin{aligned}
\mathbb{E}\left(N^{p}\left(Y_{n}\right)\right) & =\sum_{k=1}^{\infty}\left[\sum_{j=k_{n-1}+1}^{k_{n}} \int_{\mathbb{R}} \mathbb{P}\left(l_{i}<\xi_{i}(j \tau)<t\right) d \mathbb{P}\left(t_{s f}<t\right)\right] \\
& +\mathbb{P}\left(t_{s f}>\max \left\{\left(k_{n-1}+1\right) \tau-T_{n-1}+\xi_{i}\left(Y_{n-1}\right), l_{i}\right)\right. \\
& \left.+\mathbb{P}\left(t_{s f}>\max \left\{T_{n}-k \tau+\xi_{i}\left(k_{n} \tau\right), l_{i}\right\}\right)\right] \mathbb{P}\left(k_{n}=k\right) \\
\mathbb{E}\left(C_{i m}^{s}\left(T_{n}\right)\right) & =\sum_{k=1}^{n} \mathbb{E}\left(N_{k}^{f}\right)\left(C_{s f}+C_{l}\right)+\mathbb{E}\left(N_{k}^{p}\right) C_{s p}+k_{n} C_{i s}
\end{aligned}
$$

